\title{
Visual Topography in Primate V2: Multiple Representation across Functional Stripes
}

\author{
Anna W. Roe and Daniel Y. Ts'o \\ Division of Neuroscience, Baylor College of Medicine, Houston, Texas 77030
}

The second visual cortical area (V2) of the primate is composed of repeating thin, pale, and thick cytochrome oxidase stripes containing primarily color-selective, broadband oriented, and disparity-selective cells, respectively. We have now examined topography in V2 with respect to these functional subdivisions. Our data suggest that there are multiple, interleaved visual maps in V2, one for each of the color, orientation, and disparity domains. The same region of visual space is re-represented by each stripe within a stripe cycle, resulting in discontinuities or "jumps back" in representation at stripe borders. Adjacent stripe cycles represent adjacent regions of space such that the visual map is continuous from one stripe to the next like stripe. Receptive field size and scatter are significantly larger for thin stripes than for thick stripes. Unexpectedly, our data suggest two types of pale stripes within each stripe cycle, one with scatter similar to thin stripes and another to thick stripes. Some evidence also suggests the presence of multiple maps within individual stripes in V2. Consistent with functional clustering within single stripes (Ts'o et al., 1990b), we have recorded re-representations and topographic discontinuities coincident with functional borders within single stripes. These results suggest that multiple and interleaved mapping may be a common organizational strategy for representing multiple functional domains within a single cortical area.

[Key words: visual area 2, topography, cortical maps, cortical magnification, point set, point image, hypercolumn, optical imaging, cytochrome oxidase stripes]

Classically, one important criterion for defining a cortical area, whether sensory or motor, has been the demonstration of a single topographic representation within that area. Previous studies have shown that the mapping of the visual field within a cortical area displays continuity, or gradual shifts, in receptive field location. Departures from this gradual progression, such as reversals and discontinuities, often occur at boundaries between separate cortical areas. Also characteristic of areal boundaries are changes in receptive field properties, in cortical magnification,

\footnotetext{
Received Aug. 15, 1994; revised Dec. 7, 1994; accepted Dec. 14, 1994.

We thank John Maunsell for invaluable discussions and suggestions for data analysis; Geoffrey Ghose, Jonathan Horton, and Jon Kaas for helpful comments on the manuscript; and Lara Hinderstein and Carmela LoRusso for excellent technical support. This work was supported by EY06347, Whitaker Foundation.

Correspondence should be addressed to Anna W. Roe, Division of Neuroscience, S-553, One Baylor Plaza, Baylor College of Medicine, Houston, TX 77030.

Copyright (C) 1995 Society for Neuroscience 0270-6474/95/153689-27\$05.00/0
}

and in scatter (a measure of the orderliness of receptive field progression). Together with cytoarchitectonic and anatomical connectivity criteria, topographic organization has guided the delineation of cortical areas, especially within the visual system, where cortical areas abound (for reviews, see Van Essen, 1985; Kaas, 1987). More recently, developments in our understanding of cortical organization have uncovered the presence of multiple functional domains within single cortical areas.

The advent of the cytochrome oxidase staining technique and subsequent functional studies revealed the striking segregation of functional domains within the first (V1) and second (V2) visual areas in the primate. In V1, the lattice of blobs (the darkly staining spots within V1) and the interblobs (the pale staining regions between the blobs) (Horton and Hubel, 1981) are thought to be involved with the processing of color and form, respectively (Livingstone and Hubel, 1984; Ts'o and Gilbert, 1988). V2 contains a repeating stripe organization, consisting of darkly staining thin and thick stripes separated by pale stripes. These stripe compartments-the thin, pale, and thick-are large (on the order of 1 minn wide) and few (there are only about 1012 stripe cycles in dorsal V2, personal observation; J. Horton, personal communication; cf. Tootell et al., 1983; Krubitzer and Kaas, 1990), and so comprise large subdivisions of V2. They have distinct cortical (Livingstone and Hubel, 1984; DeYoe and Van Essen, 1985; Shipp and Zeki, 1985) and subcortical (Horton, 1984; Livingstone and Hubel, 1987a) connections as well as distinct functional properties (DeYoe and Van Essen, 1985; Hubel and Livingstone, 1987; Tootell and Hamilton, 1989; Ts'o et al., 1990b; Peterhans and Von der Heydt, 1993; Levitt et al., 1994). Thin stripes are characterized by a predominance of nonoriented color-selective cells, pale stripes oriented broad-band cells, and thick stripes disparity-tuned cells. They have thus been implicated in color, form, and disparity processing, respectively (e.g., Cavanagh, 1987; Livingstone and Hubel, 1987b; DeYoe and Van Essen, 1988). In this article, we have addressed how this functional division relates to topographic organization.

Previous studies on the general topography in primate V2, prior to the knowledge of the stripe organization in V2, have shown that the visual representation in V2 is split at the horizontal meridian (Allman and Kaas, 1974; Gattass et al., 1981; Weller and Kaas, 1983). That is, the superior and inferior visual fields are represented noncontiguously by ventral and dorsal V2, respectively. The result of such a mapping (see Fig. 1) is that the posterior border (at the V1/V2 border) and the anterior border (which lies in the lunate sulcus) of dorsal V2 represent the vertical meridian (VM) and horizontal meridians (HM), respectively. Thus, in the anteroposterior axis of V2 run lines of isoeccentricity (dotted lines), while in the axis parallel to the $\mathrm{Vl}$ / 

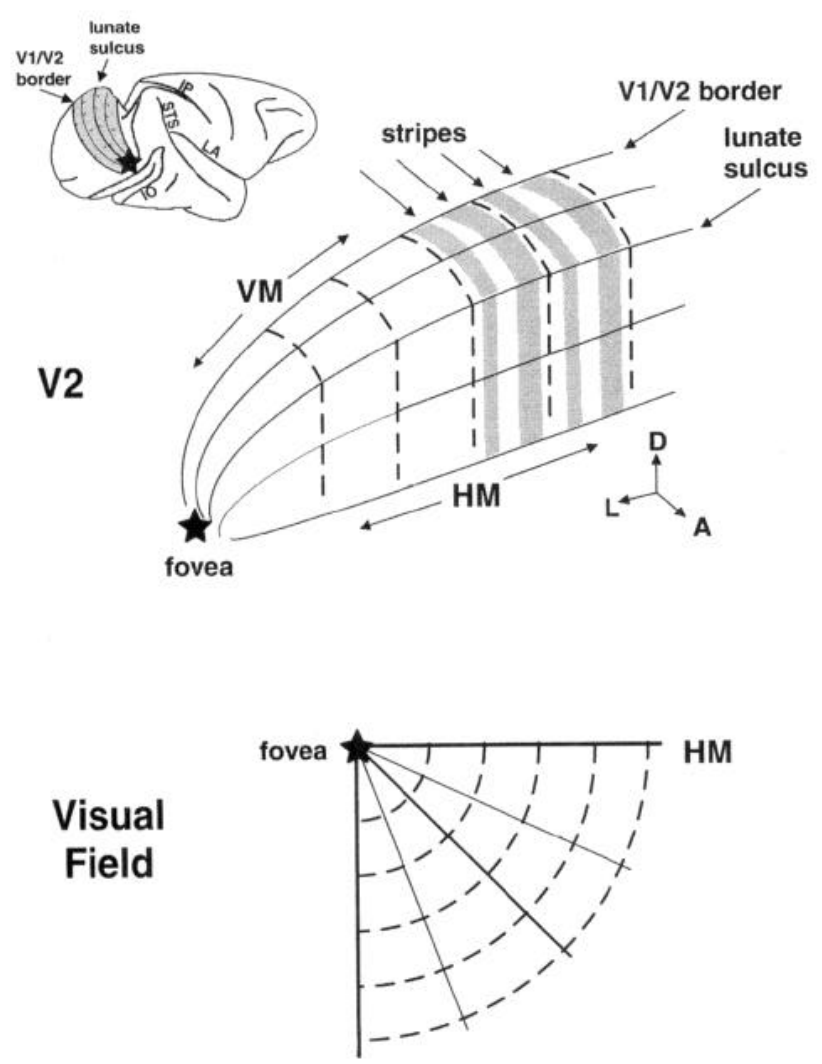

VM

Figure 1. Schematic of visual representation in dorsal V2. V2 is located on the caudal bank of the lunate sulcus and extends onto the operculum in the macaque brain (shaded region in upper left inset). An expanded view of this region (above) illustrates the representation of the visual field (below) in V2. Representation of the vertical meridian $(V M)$ lies on the V1/V2 border and that of the horizontal meridian $(H M)$ lies in the depths of the lunate sulcus. Lines of isoeccentricity (dotted lines) run perpendicular to the V1/V2 border. Lines of isopolarity (solid lines) run roughly parallel to the $\mathrm{V} 1 / \mathrm{V} 2$ border and converge at the foveal representation (star) which is located laterally on the brain surface. The orientation of stripes in V2 is indicated (gray stripes).

V2 border run lines of isopolarity (solid lines). Since stripes in V2 are oriented anteroposteriorly (Fig. 1, gray stripes), a general expectation then is that isoeccentricity lines would lie parallel to the stripes and lines of isopolarity would run across the stripes.

Figure 2 depicts two possible models of how the visual map may be represented across the functionally discrete stripes in $\mathrm{V} 2$. One possibility is that there is a single continuous representation across the stripes in V2 (Fig. 2, continuous). In this model, contiguous points in the visual field always have contiguous representations in the cortex. This would predict either functional "gaps" in visual representation within any single functional domain (e.g., lack of thin stripe representation in the portion of the visual field between two gray arrows), or, to avoid such gaps, the presence of large receptive fields (large enough to bridge the region between the two gray arrows) and/or large receptive field scatter.

A second possibility (Fig. 2, discontinuous) is that the visual field is represented in a multiple, discontinuous manner. In this model, a single region of visual space would be represented three times, once in each stripe type. Thus, an electrode recording across a thin stripe (gray arrow in V2) would encounter a
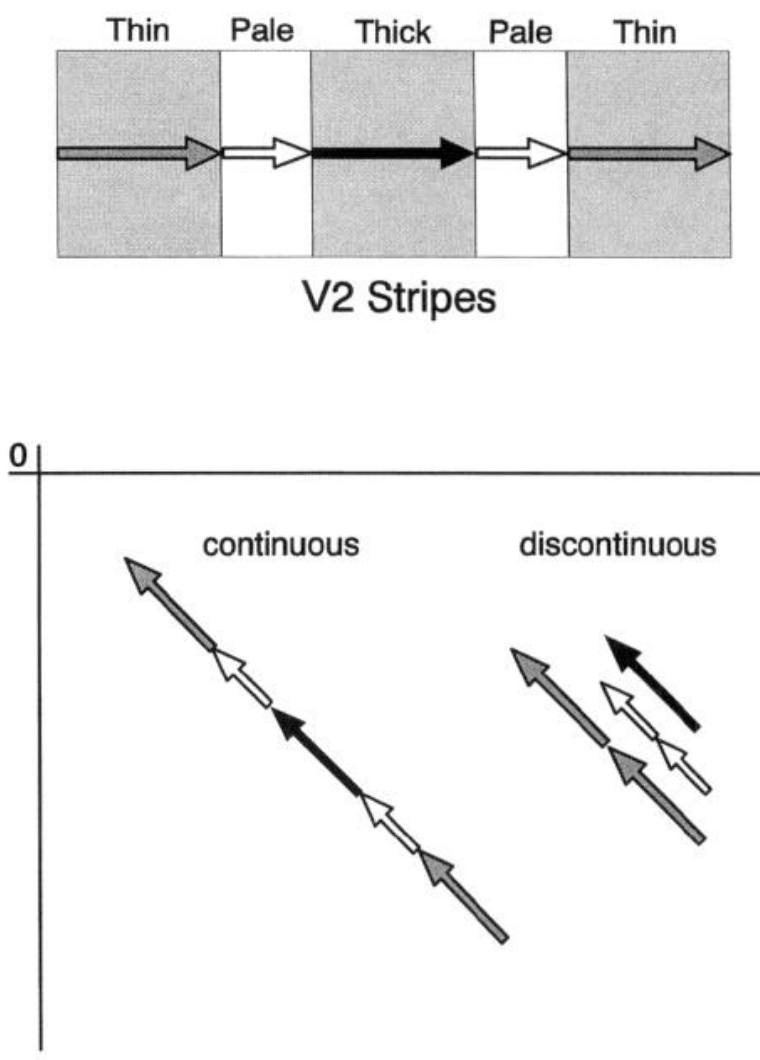

\section{Visual Field}

Figure 2. Possible mappings of the visual field in V2 stripes. A little over one cycle of thin/pale/thick/pale stripes in V2 is depicted above. Below are depicted a continuous model (left) and a discontinuous model (right) of visual representation. In the continuous model, contiguous points in the visual field have contiguous representations in the cortex, regardless of stripe location. Receptive fields would need to be quite large to span the gaps in representation in any single functional domain (e.g., from gray arrow to gray arrow in the visual field). In the discontinuous model, representation, while continuous within any single stripe, would be discontinuous at stripe borders. Thus, any single region of visual space would be triply represented (by one gray, two white, and one solid arrow in the visual field). Continuity within any functional domain would extend from one stripe to the next functionally similar stripe (e.g., from gray arrow to gray arrow in the visual field).

sequence of color cells whose receptive fields shift in a continuous progression in the visual field (lower gray arrow in the visual field below). At the thin/pale border, there would be a jump back in visual representation (to beginning of white arrow), followed by a progression of oriented broad-band cells in the pale stripe. At the pale/thin border, there would be another jump back in the visual field (to beginning of black arrow), followed by a series of disparity-selective cells. Three predictions emerge from this second model. It predicts that any region of visual space is multiply represented, once in each of the three functional stripes. It predicts the presence of topographic discontinuities at stripe borders. And, in order to attain a complete visual map in any single functional domain, it predicts visuotopic continuity from one stripe to the next like stripe (e.g., gray arrow to gray arrow).

This type of multiple representation was initially suggested by Hubel et al. (1974) with respect to ocular dominance columns in layer IVC of V1 and by Zeki and Shipp (1987) with respect to stripes in V2. In this article, we have investigated the topog- 
raphy of V2 with respect to functional compartments by recording across V2 stripes with long tangential electrode penetrations. To more precisely identify stripe boundaries, we have used optical inlaging methods and characterization of cells based on color, orientation, and disparity preference. Our findings fulfill each of the predictions of the second model of representation. In addition, we find evidence for further topographic divisions within individual stripes.

Some of these results have been previously presented (Roe and Ts'o, 1992, 1993a,b; Ts'o and Roe, 1994).

\section{Materials and Methods}

\section{Surgical preparation}

Eight hemispheres in seven adult monkeys (Macaca fascicularis) were used for these mapping experiments. Four of these monkeys were also used for other physiological experiments (cf. Roe and Ts'o, 1992; Ts'o et al., 1993). Following an initial anesthetic dose of ketamine hydrochloride $(10 \mathrm{mg} / \mathrm{kg})$, animals were intubated endotracheally and a catheter implanted in the saphenous vein for drug delivery. Anesthesia was maintained throughout the experiment by a constant infusion of sodium thiopental $(2 \mathrm{mg} / \mathrm{kg} / \mathrm{hr}$ ). Animals were paralyzed (vecuronium bromide, $100 \mu \mathrm{g} / \mathrm{kg} / \mathrm{hr}$ ) and respirated. Vital signs including heart rate and EEG were continuously monitored; rectal temperature was maintained at $38^{\circ} \mathrm{C}$ and expired $\mathrm{CO}_{2}$ at $4 \%$. $\Lambda$ fter dilation of the pupils (atropinc sulfate $1 \%$ ), the eyes were fitted with appropriate contact lens to focus on a tangent plane 57 inches in front of the animal. The foveas were projected onto the tangent screen with a fundus camera. A $1 \mathrm{~cm}$ craniotomy and durotomy were made over a region around the lunate sulcus (centered about $15 \mathrm{~mm}$ anterior to occipital ridge and $10 \mathrm{~mm}$ lateral to midline). This exposed visual cortex near the V1/V2 border represents the visual field between $2^{\circ}$ and $5^{\circ}$ eccentricity. The eyes were then converged by placing a Risley prism in front of one eye and achieving precise overlap of right and left eye receptive fields of a V1 or V2 binocular cell.

\section{Optical imaging}

Prior to electrophysiological recording, we obtained functional maps of cortical organization by optical imaging of intrinsic cortical signals (Ts'o et al., 1990; cf. Grinvald et al., 1986, 1988). This is a technique which detects activity-related reflectance changes in response to different visual stimulation conditions. We routinely generated functional maps for ocular dominance, orientation, color, blob/interblob patterns in V1, and stripe locations in V2 (Ts'o et al., 1990). By revealing cortical organizations relative to cortical surface vasculature, this method allowed precise targeting of cortical structures for purposes of electrophysiological recording with microelectrodes or for tracer injections. These maps could be used for multiple recording sessions within the same cortical region. For increased cortical stabilization during optical recording, an optical chamber was cemented over the craniotomy, filled with lightweight silicone oil, and sealed with a cover glass. The cortical surface was illuminated through the chamber window with $630 \mathrm{~nm}$ wavelength light provided by optic fiber light guides. A slow-scan CCD camera (Photometrics) fitted with standard $35 \mathrm{~mm}$ camera lenses was then positioned over the chamber.

Images of the cortical surface were collected during visual stimulation and, in order to minimize movement noise due to respiration and heartbeat, frame collection was synchronized in phase with respiratory and heart cycles. All visual stimuli were presented with a Barco color monitor controlled by an IBM PC/AT with a Number Nine graphics card. A variety of visual stimuli, including luminance and chromatic contrast gratings, stationary and moving, of different spatial frequencies and orientations were presented in a pseudorandom fashion. For color stimuli, the monitor was calibrated to present isoluminant color contrast gratings. An electromechanical shutter in front of each eye allowed for independent stimulation of each eye. Images were digitized, collected, and processed by a DEC Microvax III computer. Typically in a single trial, 10 successive frames were collected per stimulus condition over a period of 2-3 sec. Stimulus conditions (out of either 16 or 32 total) were presented in an interleaved, pseudorandom fashion. All trials (usually 16) acquired for each stimulus condition were summed and divided by the sum of blank stimulus trials. This procedurc maximizes signalto-noise ratios and minimizes effects of uneven illumination. For each functional property (e.g., ocular dominance, orientation, color/luminance preference), the sum of images obtained under one stimulus condition (e.g., left eye) were subtracted from that obtained under another (e.g., right eye). These difference images were then clipped, scaled, and smoothed, and displayed on an RGB color monitor or printed out for further inspection and comparison.

\section{Electrophysiological recording}

Tungsten microelectrodes (Ainsworth) were angled tangentially $\left(\sim 30^{\circ}\right)$ relative to the cortical surface and were advanced either across or along V2 stripes (as identified by optical imaging). Units were recorded every $100 \mu \mathrm{m}$ along the penetration until white matter was reached, thus allowing for successive recording of about 40 units along a single penetration. Since the widths of V2 stripes are a millimeter or so, we chose $100 \mu \mathrm{m}$ as a constant sampling distance. This allows both a reasonable sample size within single stripes as well as the ability to sample across the distance of several stripes within a single penetration. The receptive field location of a reference cell recorded in VI was measured relative to the foveal location, as determined by back-projection onto the tangent screen with a fundus camera. To account for possible drift in eye position, the location of this reference cell was replotted after recording every V2 unit. All V2 receptive field positions were measured relative to the receptive field location of this reference cell.

To characterize cells, receptive fields were plotted on the tangent screen with a hand-held projection lamp. To avoid errors in receptive field position due to adjustments in prism settings (for characterization of disparity-selectivity), receptive fields were all plotted through the eye without the prism, except in cases of obligatory binocular cells in which reccptive fields were plotted binocularly. We characterized each unit for ocular dominance, peak and width of orientation tuning, degree of direction selectivity, and degree of end inhibition. Orientation selectivity was rated on a qualitative scale $\mathrm{A}-\mathrm{D}$, where $\mathrm{A}$ is most narrowly $\left(<30^{\circ}\right)$ and D is most broadly tuned (cf. Livingstone and Hubel, 1984); cells rated A or B were considered oriented. Color selectivities were determined by using narrow band interference filters equalized for radiance, ranging from $450-630 \mathrm{~nm}$ in $30 \mathrm{~nm}$ increments. Nonoriented color cells without antagonistic surrounds were classified as type II and those with broad-band antagonistic surrounds were classified as modified type II (Ts'o and Gilbert, 1988). Occasionally true double-opponent cells were encountered (Livingstone and Hubel, 1984). Cells with broad-band center-surround organization were classified as type III. Cells which responded strongly to tiny stimuli within the receptive field but poorly to stimulation of the entire receptive field were classified as spot cells (Baizer et al., 1977; Hubel and Livingstone, 1987). Disparity tuning was determined by changing prism settings in $0.125^{\circ}$ steps and was rated on a scale of $0-3$ for relative response of binocular to monocular stimulation. Obligatory binocular cells, which are silent under monocular stimulation, but respond briskly under disparity-specific binocular conditions, were often encountered in thick stripes (Hubel and Wiesel, 1970; Ts'o et al., 1991). Mixed property cells (such as color disparity cells, mixed color cells, color oriented cells) were also observed. For more further description of our characterization of $\mathrm{V} 2$ receptive field properties, see Results and Ts'o et al. (1990b)

In some experiments, pressure injections of red or green fluorescent latex beads (Lumafluor) were made with a glass pipette. In nonterminal experiments, artificial dura was implanted subdurally, the existing dura sutured closed, the bonc replaced, and the animals allowed to recover. Analgesics and antibiotics were administered upon recovery. In terminal experiments, during the recording session electrolytic lesions were made along each penetration by passing current $(4 \mu \mathrm{A}$ for $4 \mathrm{sec}$ ) through the electrode tip. At the end of data collection, animals were then given a lethal dose of sodium pentobarbital and perfused through the heart with $4 \%$ paraformaldehyde. Following removal of the brain, the relevant cortical region was removed, flattened, and sunk in $30 \%$ sucrose solution. The cortical tissue was then sectioned tangentially at $30 \mathrm{~mm}$ and alternate sections reacted for cytochrome oxidase histochemistry and coverslipped for visualization of fluorescent bead labeling. Recording sites were subsequently reconstructed and aligned with optical images using lesions, tracer injection sites, cytochrome oxidase landmarks, and blood vessels as guides.

\section{Data analysis}

Cortical magnification factor. The angle $(A)$ of the tangential penetration to the cortical surface was estimated by guide marks on the elec- 

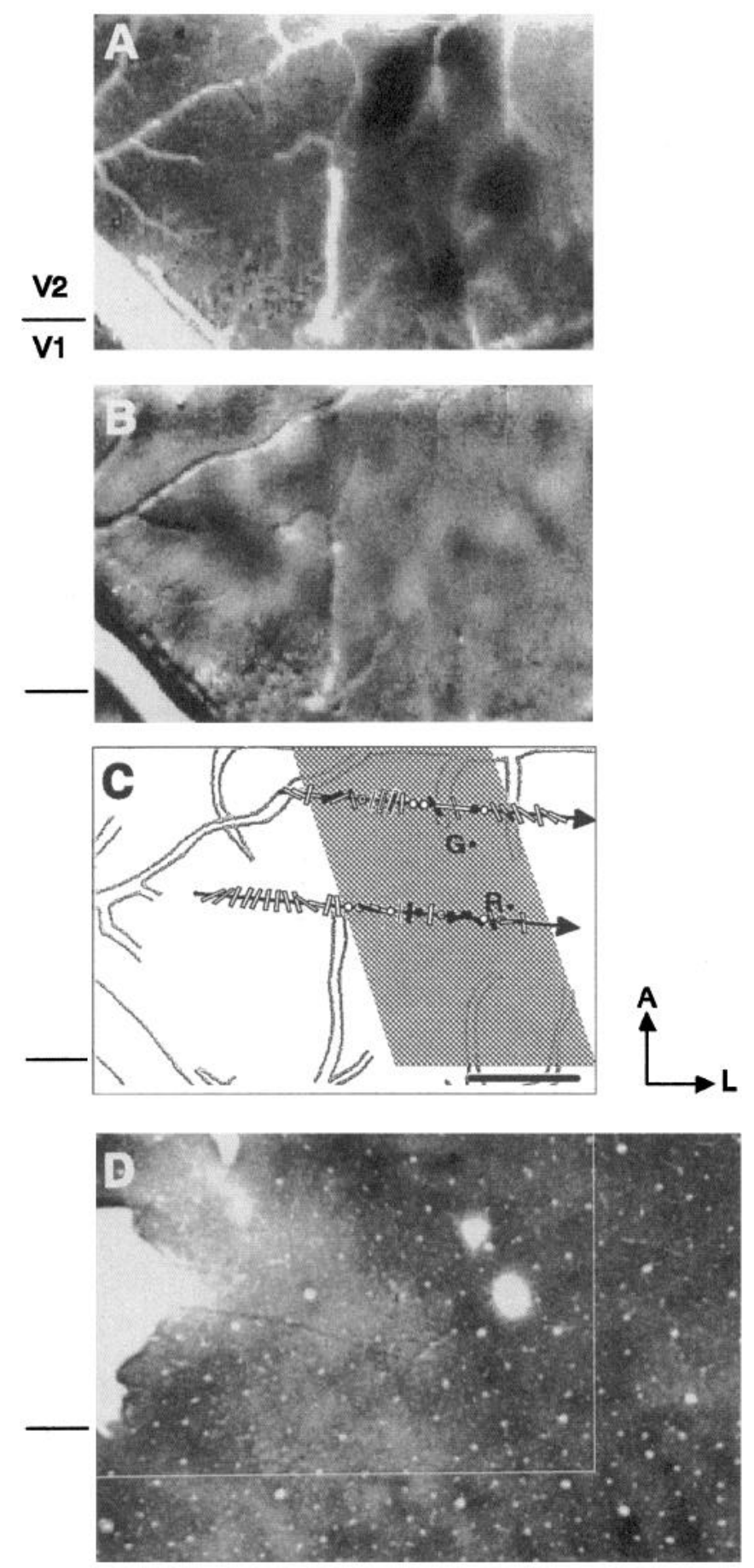

Figure 3. Optical imaging of stripes in V2. A, Color stripe in V2: an optical image of color versus luminance selective regions in V2. Darker regions within the color stripe (stripe location indicated by hatching in C) indicate those regions preferring isoluminant color gratings and lighter regions those preferring achromatic luminance gratings. Regions outside of the color stripe do not contain organization for color versus luminance activation (relatively even gray). This prominent stripe of color versus luminance activation shows substructure typical of color stripes in V2. Cortical surface vasculature appears white (e.g., a large vessel in lower left corner) and is outlined in $C$. The location of the $\mathrm{V} 1 / \mathrm{V} 2$ border is indicated by the bar at the left. Anterior is toward the top; lateral toward the right. $B$, Pale stripe in V2: an optical image of orientation selectivity in the identical cortical region shown in $A$. Dark regions indicate regions most highly activated by $90^{\circ}$ oriented gratings, while light regions indicate those preferring $0^{\circ}$ oriented gratings. There is a prominent stripe of orientation selectivity in the left portion of the trode micromanipulator. Tangential cortical distance traversed was estimated by multiplying penetration distance, as confirmed by electrolytic lesions, by $\cos (A)$. Linear cortical magnification factors $(\mathrm{mm} /$ degree) were calculated both by (1) dividing the cortical distance across the entire stripe by the visual distance between the most extreme receptive field centers along the axis of progression and (2) by taking the inverse slope of receptive field progression plots (see Fig. 8). For comparison of cortical magnifications along vs across stripes, we divided the tangential cortical distance between two cells by the distance between their receptive field locations (see Fig. 22).

Receptive field size and scatter. Receptive field size was taken as the square root of the area of the receptive field. We used as a metric for scatter the visual distance between two units recorded successively within an electrode penetration. Since recordings often spanned a few degrees of visual space, it was important for the purposes of making comparisons between stripes to normalize for changes in receptive field size or scatter due to changes in eccentricity. Therefore, for each unit we normalized receptive field size by visual eccentricity, and visual scatter by the average of visual eccentricities between the two successive units. The mean normalized receptive field size and scatter within each stripe was then calculated and compared with other stripes. Statistical comparisons were conducted with the Student's $t$ test.

Coverage overlap. Receptive field coverage was calculated by first projecting receptive fields onto the axis of visual progression (as determined by linear regression through all receptive field centers recorded in a single penetration). The number of receptive fields covering each point (in $0.1^{\circ}$ intervals) along this axis was then tabulated (see Fig. $6 B$ ). To express overlap in representation, two different overlap indices were calculated. For the first index, for each stripe, the extent of visual coverage common to both stripes was divided by coverage extent of that stripe. The larger of these two percentages was then taken as the overlap index. The second index expresses overlap weighted by the amount of coverage at each point. For two coverage distributions, $\mathrm{C} 1$ and $\mathrm{C} 2$, the overlap index is $\mathrm{OI}=(\mathrm{C} 1 \cdot \mathrm{C} 2) /(|\mathrm{C} 1||\mathrm{C} 2|)$.

\section{Results}

Note on terminology

The nomenclature "thin," "pale," and "thick" derive from descriptions of stripe patterns revealed by cytochrome oxidase staining. However, there remains significant degree of variability in both the widths and the regularity of their pattern across V2, particularly in the macaque (Hubel and Livingstone, 1987; DeYoe et al., 1990; personal observations). Furthermore, physiologically "thin" stripes are not consistently narrower than "thick" stripes as assessed by length of tangentially recording sequences across stripes in V2 (cf. Hubel and Livingstone, 1987; Zeki and Shipp, 1987), which calls into question the correlation between stripe width as seen in cytochrome oxidase staining and

$\leftarrow$

image (to the left of color stripe location). Pale stripes, unlike color stripes, are regions with strong organization for orientation. This complementarity of organization for orientation and color is consistently seen in our pale and color stripe images. Conventions as in $A$. C, Position of two tangential electrode penetrations made across the pale and color stripes shown in $A$ and $B$. Position of color stripe shown in $A$ is indicated by hatching. Gray lines trace the cortical surface vascular pattern. Receptive field properties of cells recorded along each penetration is indicated. Orientation selective cells are indicated by bars and nonoriented cells by circles. White symbols represent chromatically broad-band cells and color symbols represent color-selective cells. For color detail, see Figure 4. Consistent with identification of stripes by optical imaging, pale stripes contained many oriented broad-band cells and color stripes many color cells. $R$ and $G$ indicate locations of red and green fluorescent bead injections, respectively. Scale bar, $1 \mathrm{~mm}$ (applies to $A-D$ ). $A$, anterior; $L$, lateral. $D$, Cytochrome oxidase staining of a tangential section through this cortical region confirms that the color stripe revealed by imaging coincides with a dark cytochrome oxidase stripe. Blob pattern is seen in Vl. The two holes in this section located in the dark stripe indicate are fluorescent bead injection sites (corresponding with $G$ and $R$ shown in $C$ ). 
Table 1. Summary of all penetrations

\begin{tabular}{|c|c|c|c|c|c|c|}
\hline Penetration & $\begin{array}{l}\text { Stripe } \\
\text { sequence }\end{array}$ & $\begin{array}{l}\text { No. } \\
\text { stripes }\end{array}$ & $\begin{array}{l}\text { Orient } \\
\text { wrt } \\
\text { stripes }\end{array}$ & $\begin{array}{l}\text { No. } \\
\text { cells }\end{array}$ & $\begin{array}{l}\text { No. } \\
\text { cells/stripe }\end{array}$ & Figures \\
\hline 920728 & $\mathrm{bb} / \mathrm{col} / \mathrm{bb}$ & 3 & Across & 34 & $6,13,15$ & 8 \\
\hline 921001, Pen 15 & $\mathrm{bb} / \mathrm{col} / \mathrm{bb} / \mathrm{disp}$ & 4 & Across & 40 & $3,21,10,6$ & $5,6,12 D, 14$ \\
\hline 930318 , Pen 8 & $\mathrm{col} / \mathrm{bb} / \mathrm{col} / \mathrm{bb}$ & 4 & Across & 38 & $17,7,12,2$ & $15,17 b$ \\
\hline 930318, Pen 10 & $\mathrm{bb} / \mathrm{col}(\mathrm{disp}) / \mathrm{col}$ & 3 & Across & 21 & $8,6,7$ & $15,16,17 a$ \\
\hline 930405, Pen 3 & $\mathrm{bb} / \mathrm{col}$ & 2 & Across & 17 & 8,9 & \\
\hline 930405 , Pen 7 & col & 1 & Across & 14 & 14 & 13 \\
\hline 930405, Pen 9 & $\mathrm{bb} / \mathrm{col} /(\mathrm{bb}) / \mathrm{disp} / \mathrm{bb}$ & 5 & Across & 40 & $1,10,3,20,6$ & 13,19 \\
\hline 930819 , Pen 6 & $\mathrm{bb} / \mathrm{col}$ & 2 & Across & 31 & 13,18 & $3,4,8,12 C, 22$ \\
\hline 930819 , Pen 8 & $\mathrm{bb} / \mathrm{col} / \mathrm{bb}$ & 3 & Across & 27 & $2,19,6$ & $3,4,8,12 C, 22$ \\
\hline 921001, Pen 13 & $\mathrm{bb} / \mathrm{disp}$ & 2 & Along & 32 & 16,16 & 5,14 \\
\hline 930304 & $\mathrm{bb} / \mathrm{col} / \mathrm{bb}$ & 3 & Along & 37 & $5,20,12$ & $12 B$ \\
\hline 930331, Pen 12 & $\mathrm{col} / \mathrm{disp}$ & 2 & Along & 29 & 19,10 & $15,17 c$ \\
\hline 930405, Pen 5 & $\mathrm{bb} / \mathrm{col}$ & 2 & Along & 16 & 6,10 & \\
\hline 930415 & $\mathrm{bb} / \mathrm{col} / \mathrm{bb}$ & 3 & Along & 50 & $18,27,5$ & $11,12 A, 18$ \\
\hline Total number & 14 & 39 & $\begin{array}{l}9 \text { across } \\
5 \text { along }\end{array}$ & 426 & & \\
\hline
\end{tabular}

stripe width determined functionally. In this article, by both imaging and electrophysiological assessments, we have found that, although there was good correspondence between the positions of functional stripes and cytochrome oxidase stripes, stripes that contain predominantly color cells are not always thinner. That is, the width of the physiologically defined color stripe often exceeds the width of the cytochrome oxidase thin stripe and the width of the physiologically defined pale stripe is often narrower than the apparent width of the cytochrome oxidase pale stripe. Indeed, color cell sequences which traversed the full width across "thin" stripes averaged $1.4 \mathrm{~mm}$ ( 16 cells) and some were as long or longer as those of disparity cell sequences recorded across thick stripes (see Table 1, Figs. 4, 5). Since in this article we refer primarily to functionally determined stripes we will use the-terms "color," "pale," and "disparity" to refer to the three functional stripe types. We will use the terms "thin" and "thick" only when referring specifically to stripes defined by cytochrome oxidase.

\section{Determination of stripe types, locations, and boundaries}

We used optical imaging of intrinsic cortical signals (Grinvald et al., 1986, 1988; Frostig et al., 1990; Ts'o et al., 1990a) to localize stripes within V2. Figure 3 illustrates our strategy for revealing V2 stripe types and their locations prior to electrophysiological recording. In Figure $3, A$ and $B$ are optical images of the same region of cortex near the V1/V2 border. Figure $3 A$ reveals a color stripe in V2 (stripe location indicated by hatching in Fig. $3 C$ ) which contains regions strongly activated by color stimuli (dark patches). As will be discussed further in this article, V2 stripes are not homogeneous structures but contain significant degree of substructure (Horton, 1984; Tootell and Hamilton, 1989; Ts'o et al. 1989, 1990b, 1991; Wong-Riley et al., 1993). In color stripes, such substructure is often revealed by imaging for color versus luminance preference. The darker patches in Figure $3 A$ (more strongly activated by color isoluminant gratings) are separated by lighter patches (more strongly activated by achromatic luminance gratings). Regions outside of the color stripe do not contain strong organization for color versus luminance activation (relatively even gray). Color stripes can also be localized by imaging for isoluminant red-green versus blue-yellow preference (not shown). Imaging the same region of cortex for orientation preference (Fig. $3 B$ ) reveals pale and thick stripe locations. In regions complementary to the color stripe, there are patches of light and dark activation corresponding to preferential response to orthogonally oriented luminance gratings (located to the left of the location of the color stripe). These orientation domains may comprise the "orientation clusters" in pale stripes previously described both electrophysiologically and with optical imaging (Hubel and Livingstone, 1987; Ts'o et al., 1991). These regions containing strong organization for orientation selectivity correspond to the pale stripes in V2. While in this case we did not image for disparity, the disparity regions may be

Figure 4. Parallel tangential penetrations oriented across stripes. Receptive field center locations of cells recorded along the two electrode penetrations (Pen 6 and Pen 8) shown in Figure $3 C$. Illustrated at the top are the receptive field types recorded along each penetration and the positions of these recording sites with respect to the color stripe (dark gray) and pale (light gray) stripes ( $A$, anterior; $L$, lateral). Numbers indicate cell number in recording sequence. Broad-band oriented cells are indicated by white bars; color nonoriented and oriented cells by color circles and bars, respectively; and disparity cells by black bars (color-selective disparity cells are indicated with color outlines). Symbols with mixed colors indicate those color cells responsive to either red and green or blue and yellow wavelengths. Spot cells are indicated by symbols with black dots. Below, the visual field location of each receptive field center is plotted (elevation is plotted on the abscissa, azimuth on the ordinate). Receptive ficlds are numbered according to sequence along the electrode penetration (from left to right). Three arcs of isoeccentricity (the $3^{\circ}, 4^{\circ}$, and $5^{\circ}$ ) are shown. $B$, Receptive field plots of cells shown in A. Receptive fields of broad-band cells are drawn in black outline and color cells in colored outline. Rectangles and circles/ovals indicate oriented and nonoriented receptive fields, respectively. Disparity cells are coded with bold lines. Two (the $3.0^{\circ}$ and $4.0^{\circ}$ ) isoeccentricity arcs are shown. Two receptive fields are not drawn due to space limitations (Pen 6 , cell $19: 0.8^{\circ} \times 2.35^{\circ} ;$ Pen 8 , cell 1: $1.2^{\circ} \times 1.5^{\circ}$ ). 

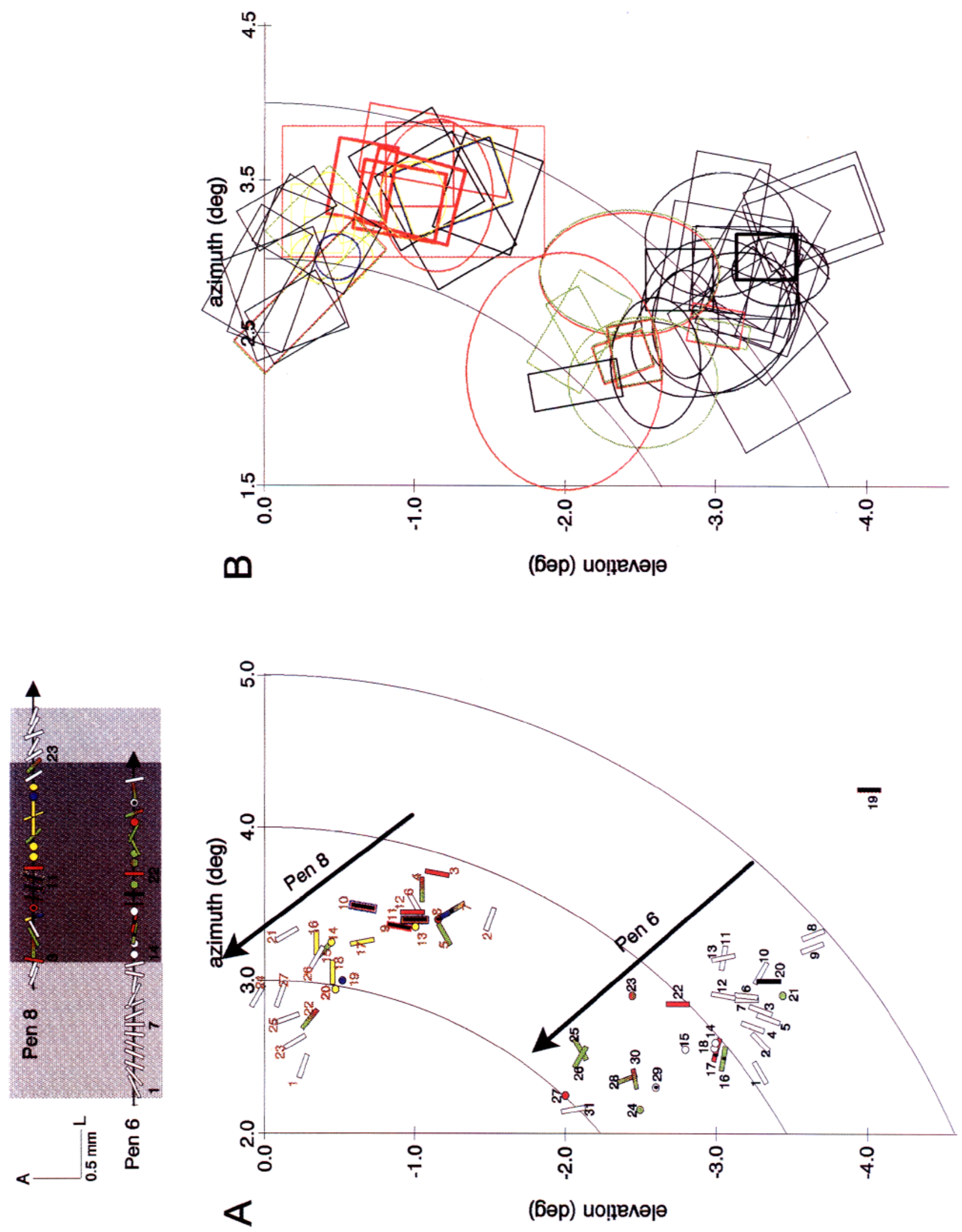

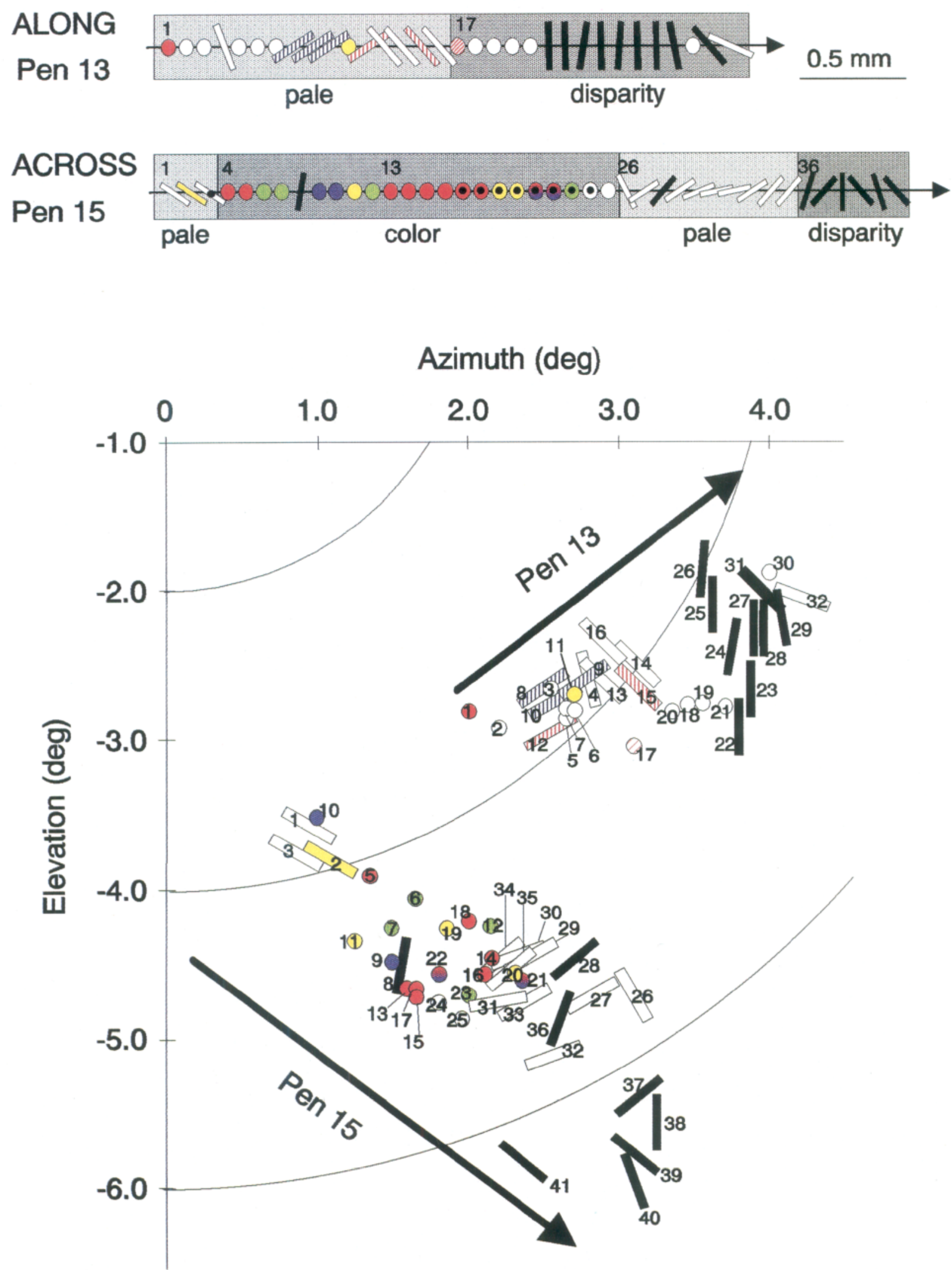

Figure 5. Orthogonal tangential penetrations, one oriented across (Pen 15) and one along (Pen 13) stripes. Above, Position of Pen 13 and Pen 15 relative to stripe borders. Pen 15 recorded part of a pale stripe, a color stripe, another pale stripe, and part of a disparity stripe. Pen 13 passed obliquely through a pale stripe followed by part of a disparity stripe. Spot cells are indicated by symbols with black dots. Striped symbols (e.g., in pale stripe of Pen 13) indicate broad-band cells with some color bias. Below, Receptive field center plots of all cells recorded in Pen 13 and Pen 15. (Cell 4 in this penetration is not plotted due to missing rf location information.) Three isoeccentricity lines (arcs) are shown ( $2^{\circ}, 4^{\circ}$, and $\left.6^{\circ}\right)$. Conventions are as in Figure 4. 
Table 2. Percentage of each cell type recorded in each stripe type

\begin{tabular}{llllllllll} 
& $\begin{array}{l}\mathrm{bb} \\
\text { orien- } \\
\text { ted }\end{array}$ & $\begin{array}{l}\mathrm{bb} \\
\text { un- } \\
\text { orient. }\end{array}$ & $\begin{array}{l}\text { Color } \\
\text { orient. }\end{array}$ & $\begin{array}{l}\text { Color } \\
\text { un- } \\
\text { orient. }\end{array}$ & $\begin{array}{l}\text { Dis- } \\
\text { parity }\end{array}$ & $\begin{array}{l}\text { Color } \\
\text { disp. }\end{array}$ & Total & Spot & $\begin{array}{l}\text { End- } \\
\text { in- } \\
\text { hib. }\end{array}$ \\
\hline Pale $(n=152)$ & 74 & 13 & 9 & 3 & 1 & 0 & 100 & 5 & 37 \\
Color $(n=232)$ & 7 & 12 & 21 & 50 & 6 & 4 & 100 & 9 & 16 \\
Disparity $(n=42)$ & 7 & 14 & 2 & 0 & 63 & 14 & 100 & 5 & 42 \\
$\quad$ Total $(n=426)$ & & & & & & & & & \\
\hline
\end{tabular}

visualized by imaging for binocular interaction (cf. Ts'o et al., 1990b, 1991).

We used images such as these to guide the placement of tangential electrode penetrations for the purpose of examining visual representation across specific stripes (Fig. 3C). Electrophysiological recordings corroborated the functional identity and locations of these stripes. In two tangential penetrations across the pale and color stripes identified in Figure $3, A$ and $B$, primarily oriented broad-band cells (white bars) were recorded in the pale stripe and primarily color cells (colored symbols) in the color stripe (Fig. 3C). Furthermore, locations of imaged stripe regions coincided well with stripes revealed by subsequent postmortem cytochrome oxidase histology (Fig. $3 D$ ), thus providing additional confirmation of the validity of the imaging technique for purposes of stripe localization in V2 (cf. Ts'o et al., 1990a).

In our experience precise localization of stripe boundaries can be uncertain with cytochrome oxidase staining alone and is greatly improved with the use of optical images and electrophysiological recordings. Thus, our division of recording sequences into so-called thin (color), thick (disparity), and pale stripes has been guided by reconstruction of electrophysiological recording sites and subsequent alignment with both optical images and cytochrome oxidase staining patterns. In virtually all cases, this led to confident assignment of recorded cells into specific stripe compartments.

\section{Receptive field properties with respect To V2 stripes}

We provide here a brief summary of the receptive field types that we observe in V2 (cf. Baizer et al., 1977; Poggio and Fischer, 1977; Hubel and Livingstone, 1987; Peterhans and von der Heydt, 1989; Ts'o et al., 1990b, 1991; Levitt et al., 1994) and, in particular, with respect to their stripe location (cf. Hubel and Livingstone, 1987; Ts'o et al., 1990b, 1991). Figures 4 and 5 illustrate correlations between receptive field types and stripe location. Further examples can be seen in Figures 11 and 1319. Percentages presented in Table 2 indicate the proportion of each cell type recorded in each stripe type, out of all cells recorded in each stripe type (color $n=232$, pale $n=152$, disparity $n=42$ ).

Our findings are in general agreement with previous studies (Hubel and Livingstone, 1987; Ts'o et al., 1990b, 1991). In pale stripes (see Table 2), by far the most commonly encountered cell type is spectrally broad-band and orientation selective (74\%). Receptive field types in color stripes are more varied, consisting primarily of color-selective cells, both oriented and unoriented. Unoriented color cells $(50 \%)$ tend to have larger receptive fields than broad-band oriented cells, while oriented color cells $(21 \%)$ often have small receptive field sizes similar to those of broadband oriented cells. "Spot cells," a higher order cell characteristic of $\mathrm{V} 2$, respond strongly to tiny (e.g., $0.125^{\circ}$ ) spots placed anywhere within a large (e.g., 2-3 $)$ receptive field, but are either inhibited by or less responsive to illumination of the entire receptive field. We encountered both nonoriented and oriented spot cells. Spot cells, in these experiments, were infrequently encountered ( $n=30$ ), but were usually color selective and located in color stripes. Some color stripes also contained clusters of disparity cells (10\%; see also Figs. 4, 16, 17). Disparity stripes are characterized by clusters of disparity cells $(76 \%)$, either predominantly broad-band or color selective (see Fig. 17), as well as occasional clusters of broad-band nonoriented cells (14\%; see Fig. 14). Obligatory binocular cells (Hubel and Wiesel, 1970) are a hallmark of V2 binocular processing, and are a predominant component of V2 disparity stripes. These cells are exquisitely selective, responding briskly only to a specific binocular disparity, often preferring vertically oriented very long and very thin bars of light, and completely silent to monocular stimulation. In general, border regions often contain cells of transitional (e.g., broad-band unoriented cells at pale/color stripe borders) or mixed functional properties, such as oriented color cells at pale/ color stripe borders or color disparity cells at color/disparity stripe borders (cf. Ts'o et al., 1990b). Color stripes contained somewhat fewer $(16 \%)$ end-inhibited cells than pale $(37 \%)$ or thick stripes $(42 \%)$.

We describe in detail the receptive field types recorded in a typical tangential penetration shown in Figure 4 (penetration 6). In this penetration which traversed a pale stripe followed by a color stripe, we recorded a sequence of sharply oriented broadband cells in the pale stripe (cells 1-12). The border between pale and color stripes in V2 is marked by a transition from cells with sharply tuned (cells 1-12) to broadly-tuned (cell 13) to those lacking (cells 14-15) in orientation selectivity. Such rapid transitions (usually within 100-200 mm) in selectivity are in general typical of stripe borders. The color stripe (cells 13-31) contains primarily color selective cells, both nonoriented and oriented, as well as two disparity cells. In this penetration, cells 19-27 were characterized by much stronger binocular than monocular responsiveness, although (with the exception of cells 19 20) without prominent disparity selectivity. Cells 16, 17, and 29 were termed spot cells because they were as responsive to tiny stimuli $\left(<0.1^{\circ}\right)$ placed anywhere in the receptive field as illumination of the entire receptive field $\left(0.75^{\circ}\right)$.

\section{Overall topography in $\mathrm{V} 2$}

In the macaque monkey V2 lies just anterior to V1 and continues in the depths of the lunate sulcus (Fig. 1). Although most of dorsal V2 lies within the lunate sulcus, a significant portion of the central $5^{\circ}$ of visual field is represented within the $2-5 \mathrm{~mm}$ strip of V2 which is exposed on the cortical surface. The bulk of the data reported here is taken from this surface V2 representing the inferior visual field between $2-5^{\circ}$ eccentricity. We 
made 14 tangential penetrations in this region of $\mathrm{V} 2$ : nine were oriented across stripes and five roughly along or skewed with respect to the stripes (see Table 1).

As described in Figure 1, the position of stripes in V2 predicts that lines of visual isoeccentricity (dotted lines) would map along the stripes, while lines of isopolarity (solid lines) would map across the stripes in V2. The following two examples confirm this expectation. In Figure 4 we plot the receptive field centers of cells recorded in the two electrode penetrations across V2 stripes shown in Figure $3 C$. Receptive fields are numbered according to recording sequence along the penetration. The general progression of receptive field centers is upward and leftward (arrows), crossing approximately orthogonal to lines of isoeccentricity $\left(3.0^{\circ}, 4.0^{\circ}\right.$, and $5.0^{\circ}$ isoeccentricities shown). This is consistent with advancing in a mediolateral direction across V2 (toward the foveal representation). [The representation of the two progressions are separated hy roughly $1.5^{\circ}$ of visual space, corresponding with a $1 \mathrm{~mm}$ cortical separation. See Mapping parameters ... cortical magnification, below.] Figure 5 is a visual field plot of cells recorded in two orthogonal penetrations in V2, one oriented predominantly across (Pen 15) and one predominantly along (Pen 13) the stripes (Fig. 5, top). As expected the receptive fields from these two penetrations progress along roughly orthogonal visual axes, one across lines of isoeccentricity (Pen 15) and one along (Pen 13).

\section{Multiple and discontinuous representation across stripes in $V 2$}

Multiple representation of visual space by functionally distinct stripes. Figures 6 and 7 demonstrate that there is a high degree of spatial overlap in the visual field representation by adjacent color, palc, and thick stripes. In Figure $6 \mathrm{~A}$ is plotted the receptive fields of cells recorded in Pen 15 of Figure 5. This penetration began in the middle of a pale stripe, passed through the full width of a color stripe, followed by another pale stripe, and part of a disparity stripe before entering white matter (Fig. 6A, top). As discussed above, color, broad-band oriented, and disparity cell types are quite segregated in the cortex: pale stripes contain primarily broad-band oriented cells, color stripes primarily color nonoriented cells, and disparity stripes disparity cells. Their receptive fields, in contrast, are considerably overlapped. For example, between $-4.0^{\circ}$ and $-5.5^{\circ}$ azimuth, there are broad-band oriented (boxes with solid lines), color nonoriented (color circles), and disparity (boxes with dotted lines) receptive fields. The extent of representation along the axis of receptive field progression is shown by the black bar (color stripe), the gray bar (pale stripe), and the dashed bar (disparity stripe).

This overlap is more quantitatively illustrated by Figure $6 B$. To compare the spatial representation from one stripe to the next, we projected each receptive field onto the axis of visual progression (as determined by a regression line through all receptive field centers) and then counted the number of cells in each stripe whose receptive fields covered each point along this axis (measured in $0.125^{\circ}$ intervals). Figure $6 B$ shows that each stripe covers roughly $2^{\circ}$ of visual space along the progression axis. Coverage by the color stripe falls between $3.5-5.9^{\circ}$ along the progression axis, that of the pale stripe hetween $4.5-6.6^{\circ}$, and that of the disparity stripe between $4.4-7.3^{\circ}$. In addition, there is an overall shift in spatial representation from one stripe to the next, a pattern which we see consistently across V2 stripes. [Note: any skewness of the electrode track relative to stripe borders will result in some shift in the visual representation from stripe to stripe. See Continuity from one stripe to next like stripe.]
Despite this shift, however, there is a substantial $1.5^{\circ}$ region of visual space commonly represented by all three stripes (indicated by bar at top). We have observed this high degree of overlap in representation in each of 10 penetrations which traversed two or more functional domains. In none of the penetrations was there a lack of overlap in visual representation from one stripe to an adjacent stripe.

To express the degree of overlap in representation between stripes, we calculated two separate indices. The first overlap index expresses the extent of visual space (along the receptive field progression axis) common to two stripes as a percentage of the stripe with narrower representation. The second overlap index is a vector dot product of the two stripe coverages (which reflects the area underneath curves in Figure $6 B$ common to two stripes). Thus, while the first index expresses overlap as the presence of any representation in common, the second takes into account the amount of representation in common at each spatial location (see Materials and Methods).

For the example illustrated in Figure $6 B$, as measured by the first index, the color and pale stripes shared $62 \%$ overlap, the pale and disparity stripes $100 \%$ overlap, and the color and disparity stripes $63 \%$ overlap. The two pale stripes exhibited $0 \%$ overlap. In our sample of 14 electrode penetrations crossing 20 stripe borders (see Fig. 7), the average degree of overlap between adjacent stripes (i.e., pale/color, pale/disparity, and one color/disparity) is $80 \%(n=17)$. The overlap between nonadjacent color/disparity stripes $(n=4)$ averaged $69 \%$. In contrast, nonadjacent pale stripes (i.e., pale stripes with an intervening color or disparity stripe, $n=4$ ) shared 6\% overlap on average. This smaller degree of overlap is statistically significant (Student's $t$ test: $p<0.0001, p<0.0003$, respectively) and is considerably lower than the value of $49 \%$ predicted by successive $80 \%$ and $69 \%$ overlaps. There is no significant difference in the $80 \%$ and $69 \%$ overlap values $(p>0.1)$. The overlap values of the second index were smaller in magnitude, but produced similar results. Average overlap for adjacent stripes was $53 \%$ and for nonadjacent color disparity stripes 44\%. Again, overlap for pale-pale stripes was statistically significantly lower (1\%).

Thus, both measures of overlap indicate that color, pale, and disparity stripes which are adjacent to each other (i.e., fall within the same stripe cycle) tend to represent the same region of visual space, while the regions of visual space represented by two stripes of similar functionality (even two pale stripes within the same stripe cycle) tend to be nonoverlapping. This finding not only suggests multiple representation by the three stripe types, but also suggests that stripes of any single functional domain (e.g., color/color) share little overlap in visual field representation. This latter point will be addressed further below (see Continuity from one stripe to the next like stripe).

Discontinuity at stripe borders. While overall progressions are easily seen over the extent of a few millimeters of cortex in V2 (Figs. 4, 5, arrows), closer inspection of individual progressions reveals that there is also a significant amount of scatter within each progression. For example, in the pale/color stripe sequence shown in Figure 4, penetration 6, cells 1-7 are separated by on average $0.1^{\circ}$ and progress not in the direction of overall progression, but follow an axis roughly orthogonal to it. Between cells 7 and 8 , there is a large jump $\left(0.6^{\circ}\right)$, followed by cells $8-$ 13 which map roughly along the overall progression axis. At the pale/thin border between cells 13 and 14, there is another large jump in progression, followed by an overall upward progression in the color stripe. 


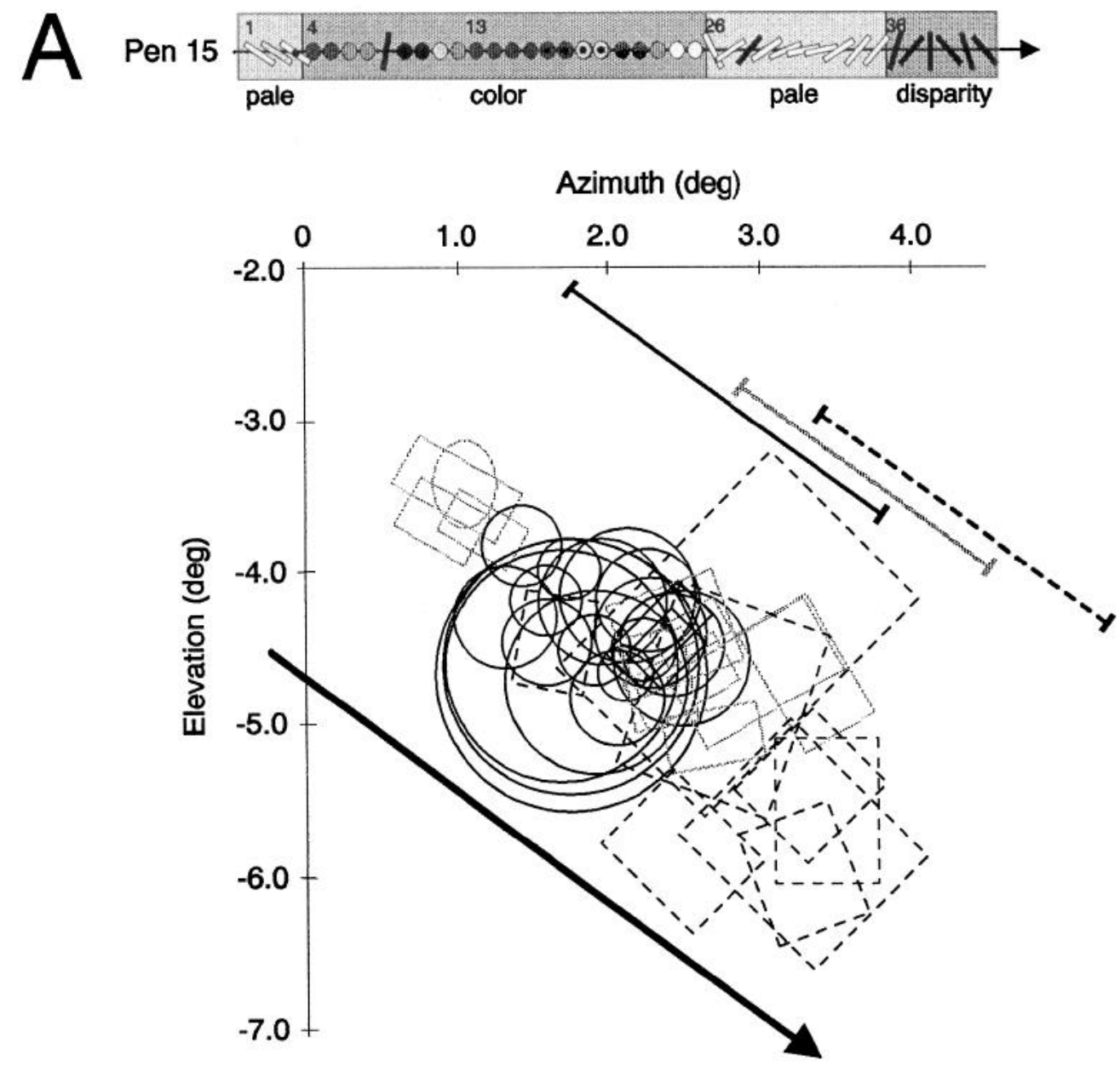

$\mathrm{B}$

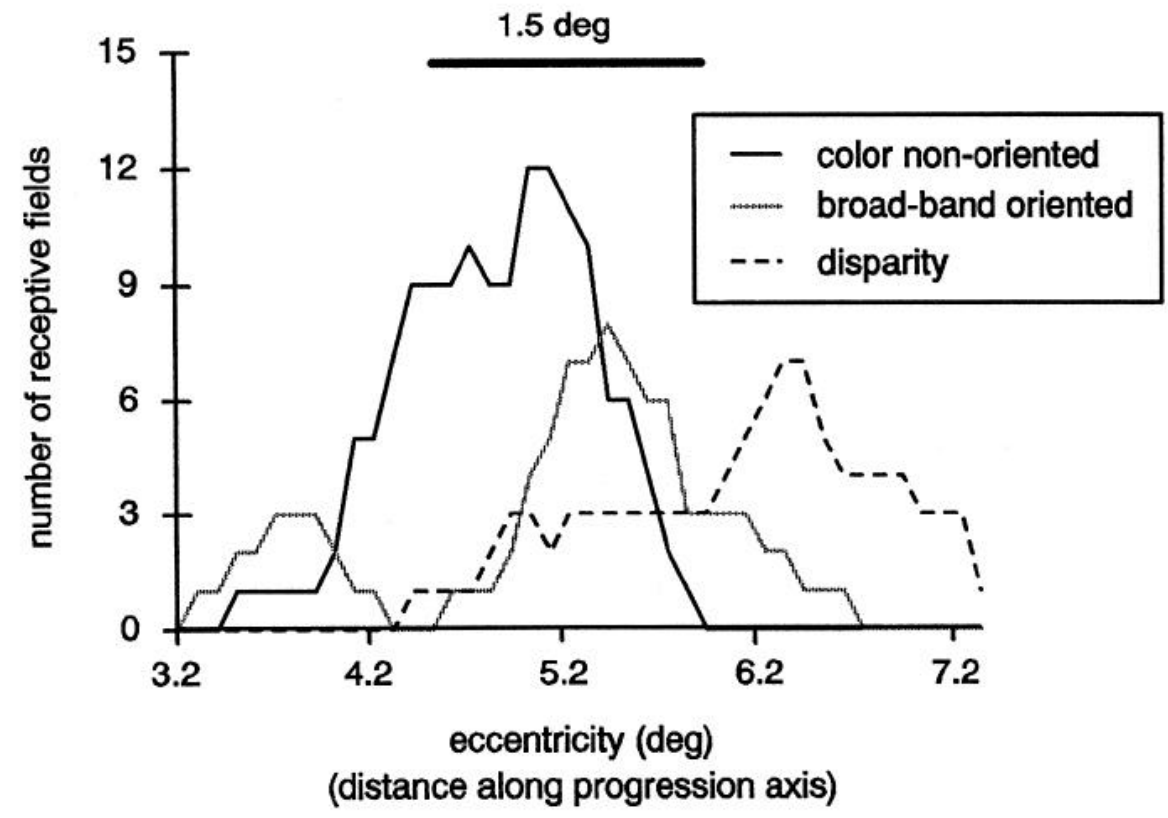

Figure 6. Re-representation across stripes. A, Receptive field plots of all cells recorded in Pen 15 from Figure 5 (shown again at top). Nonoriented color receptive fields are represented by circles, oriented cells by rectangles, and disparity cells by dashed rectangles. Arrow indicates direction of 
One way to graphically illustrate the visual progression from one recorded cell to the next is to simply plot the changes in receptive field location relative to cortical location. One would expect that, in general, the further apart two cells are in cortex, the further apart their receptive field locations will be. For a constant local cortical magnification factor, this would predict a linear relationship between cortical distance and visual distance. A poorly ordered progression would exhibit no correlation between cortical distance and visual distance. Furthermore, any abrupt changes in progression would appear as a sudden change in visual separation relative to cortical separation. Figure 8 illustrates this relationship for penetration 6 from Figure 4 .

In Figure $8 B$, we calculated the distance of each cell in penetration 6 from the beginning of the penetration (cell 1). To a first approximation, there is a positive correlation $(r=0.69)$ between cortical distance and the visual distance represented by that span of cortex. Two aspects of this graph suggest that these data are better described as two separate receptive field progressions rather than one. Separation of these points into two groups (Fig. $8 A$ ) - those falling in the pale stripe and those falling in the color stripe-improves the regression fits markedly $(r$ $=0.81$ pale stripe, $r=0.81$ color stripe). In addition, at the border between the two stripes there is a relatively large jump in receptive field progression. Within the pale stripe, the average visual distance change from one cell to the next is $0.22^{\circ}$ $\left( \pm 0.16^{\circ}\right)$; within the color stripe, it is $0.33^{\circ}\left( \pm 0.23^{\circ}\right)$. The jump in receptive field location at the border is much larger $\left(0.55^{\circ}\right)$. In fact, in 28 out of 35 comparisons receptive field jumps at stripe borders were larger than those within either the preceding or following stripe (see Fig. 9); these differences were statistically significant (paired $t$ test between average jump size within each stripe and jump at preceding border $p<0.0003$ or following border $p<0.0006$ ). [Large jumps also occur at functional borders within stripes. This issue will be further addressed below (Re-representation within single stripes).] This analysis is thus suggestive of representational continuity within stripes and discontinuity between stripes.

Continuity from one stripe to the next like stripe. The third prediction of the model concerns completeness of visual representation in any single functional domain. To achieve visual representation without gaps, one would expect continuity in representation from one stripe to the next like stripe. We have obtained evidence for stripe-to-like-stripe continuity for each of the pale, color, and disparity systems. Figure 10 illustrates schematically the expected receptive field progressions in a tangential penetration across two successive functionally similar stripes in $\mathrm{V} 2$, in this case two pale stripes. For electrode penetrations crossing stripes exactly perpendicular to stripe borders (Fig. 10, top, penetration 1), one would expect the visual field representation at the end of one pale stripe to be continued at the beginning of the next pale stripe (point A). In penetrations which are skewed relative to stripe borders, entry into the second pale stripe is shifted to point $\mathrm{B}$, resulting in an apparent gap or hole

\section{$\%$ VISUAL FIELD OVERLAP}

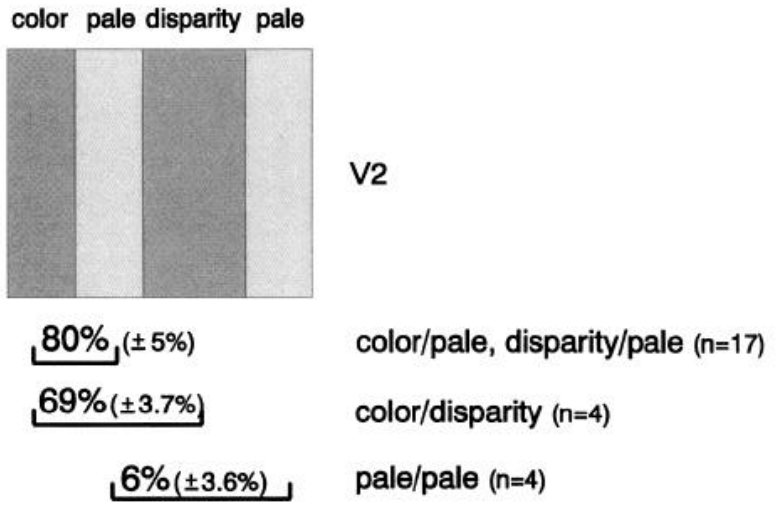

Figure 7. Percent of overlap (as measured by the first index) in visual representation by pairs of stripes within a stripe cycle. These included adjacent color/pale and disparity/pale stripes, nonadjacent color/disparity stripes within a stripe cycle, and pairs of pale stripes separated by an intervening color or disparity stripe. SEs are indicated in parentheses. Only stripes which were fully or nearly fully traversed are included in this figure. Functionally different stripes within a single stripe cycle, whether adjacent or nonadjacent, demonstrate a high degree of overlap in visual field representation. In comparison, stripes of similar functionality show very little receptive field overlap. Thus, within a stripe cycle, the color, pale, and disparity stripes rerepresent the same region of visual space, while two successive pale stripes represent distinct but adjacent points of visual space. Continuity between successive color stripes and successive disparity stripes is addressed in Figures 11-14.

in representation (between point $\mathrm{A}$ and point $\mathrm{B}$ ). Points $\mathrm{A}$ and B fall along different lines of isopolarity, but share the same isoeccentricity representation. These shifted patterns were commonly seen between the visual field map of two successive pale stripes.

Figure 11 illustrates one such example which traversed a palecolor-pale sequence (Fig. 11 $\mathrm{A}$, gray-black-gray arrows). This penetration, which was skewed with respect to the stripe borders, resulted in an apparent gap between the receptive field centers in one pale stripe and the second pale stripe (Fig. $11 \mathrm{~B}$, dotted boxes). Consistent with the orientation of the penetration, this gap is due entirely to a shift along a line of isoeccentricity. The first pale stripe receptive field centers (Pale1) fall between eccentricities of $3.5-4.5$; those in the second stripe (Pale2) fall between 3.1-3.6 eccentricities (Fig. 11B). Thus, the second pale stripe picks up where the first left off (i.e., around eccentricity 3.5). Figure $11 C$ plots the receptive fields recorded in each of these two pale stripes. Note that except for one large receptive field (\#47), there is very little overlap between the visual space covered by these successive pale stripes. To eliminate the contribution of the skew, we assumed constant isopolarity and calculated coverage with respect only to changing eccentricity.

The results of this calculation are shown in Figure $12 A$. De-

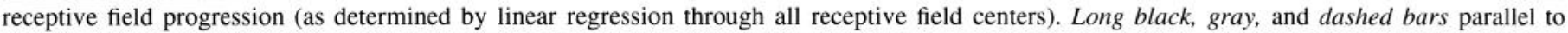

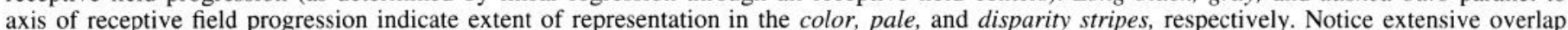

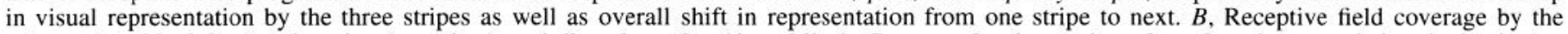

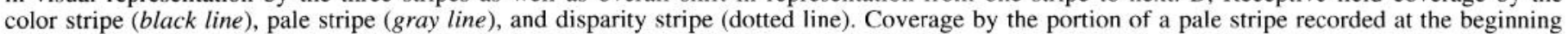

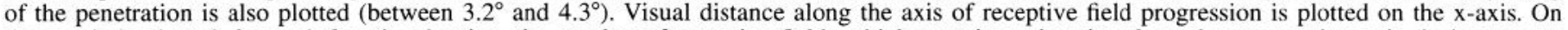

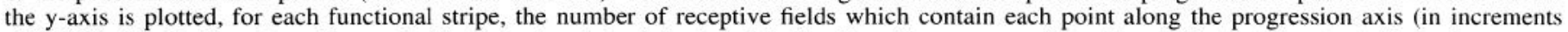

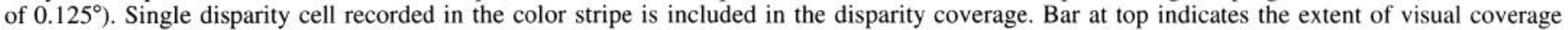
common to three successive color, pale, and disparity stripes. 


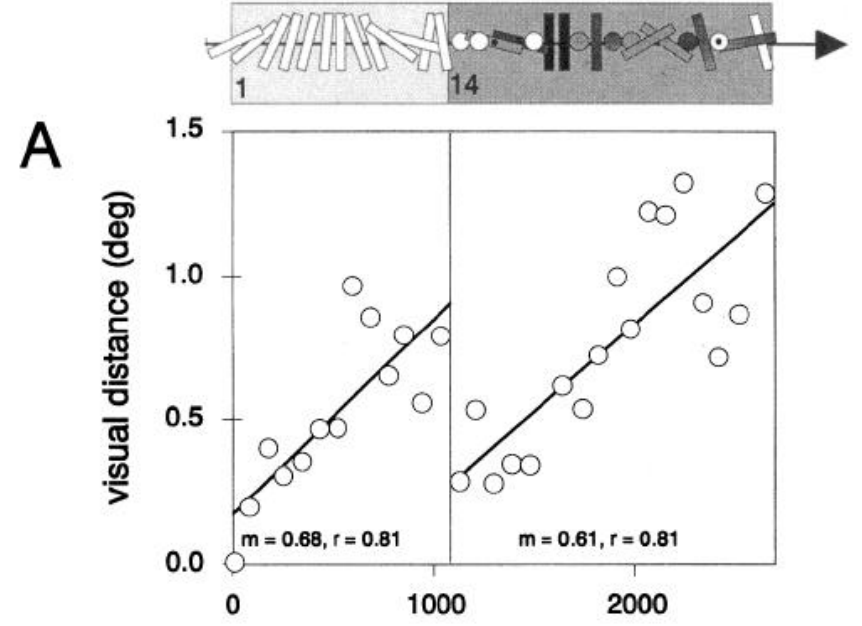

B

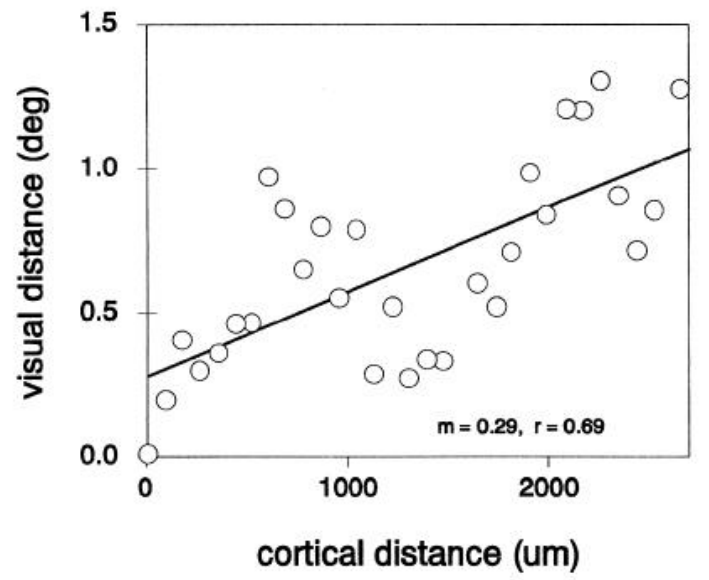

Figure 8. Discontinuity at stripe borders. Cortical distance versus visual distance plots for a pale stripe/color stripe penetration (Pen 6 shown in Fig. 4). Cortical distances were calculated assuming a $30^{\circ}$ electrode angle relative to cortical surface. The same set of points are plotted in $A$ and $B$. On the $\mathrm{x}$-axis is plotted the cortical distance in millimeters of each recorded unit from the first unit recorded in the penetration. The visual distance (in degrees) of each receptive field from the first receptive field recorded in the penetration is plotted on the $y$-axis. Points are fit by linear regression (slopes, $m$, and regression coefficients, $r$, shown). The data are considered as a single progression in B (regression coefficient $r=0.69)$ and as two progressions in $A(r=0.81$ in pale stripe, $r=0.81$ in color stripe). This analysis suggests that these data are better interpreted as two progressions separated by a discontinuity at the pale/ color stripe border than as a single progression. Due to its eccentric location with respect to the rest of the receptive field progression, the visual distance value of cell $19\left(=2.0^{\circ}\right)$ is not shown and has been excluded from the regression calculation.

spite the apparent gap, there is actually significant overlap $\left(1.1^{\circ}\right)$ in representation with respect to eccentricity between the two pale stripes. Other examples are shown in Figure $12 B-D$. Figure $12 B$ is a case in which two successive pale stripes were recorded in a penetration oriented nearly parallel to the stripes. Note that only a portion of each pale stripe was recorded, thus resulting in a smaller total extent of coverage (about $1^{\circ}$ ). In one case (see Fig. 4) in which we observed an apparent lack of (or sparsity of) pale stripe representation when examined with respect to eccentricity (Fig. 12C), there was an unusual number of color oriented cells in the intervening color stripe. This raises the possibility that the color oriented system may at times serve a similar function as (i.e., fill in the gaps for) the orientation system.

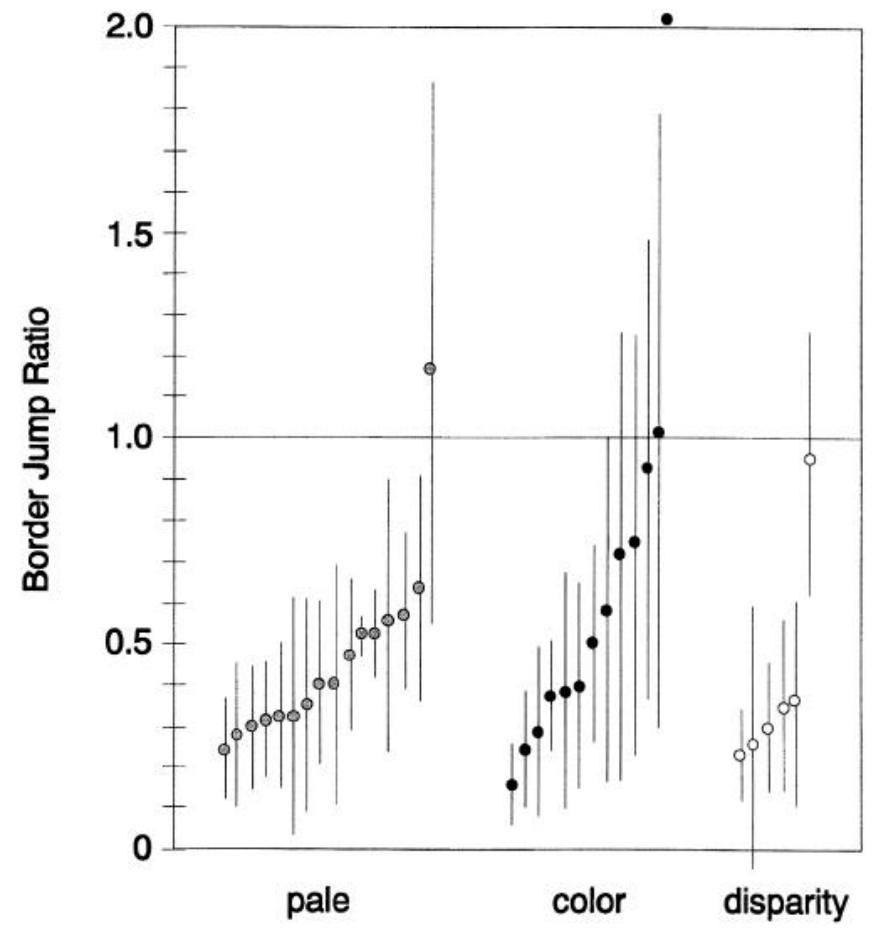

Figure 9. Receptive field jump sizes are significantly greater at stripe borders than the average jump values within stripes in 28 out of 35 comparisons. Solid symbols (pale, gray; color, black; disparity, white) indicate average jump size between successive receptive fields within an individual stripe, normalized to the jump size at the following or preceding (since only portions of some stripes were recorded) border. SDs are indicated by vertical bars. When within-stripe and at-border values are compared in a paired $t$ test, this difference is significant to $p<0.0006$ ). One color stripe (see Fig. $17 B$ ) had an unusually small border jump $\left(0.11^{\circ}\right)$, thereby resulting in a large jump ratio $\left(3.67^{\circ} \pm\right.$ $2.56^{\circ}$, plotted off the scale at the top of the graph).

Figure $12 D$ illustrates a quite noticeable sparseness of pale stripe representation in the region near the $4.2^{\circ}$ eccentricity. However, we consider it unlikely that this reflects a true lack of representation by the pale system because of the receptive field sizes and scatter in V2. The aggregate receptive field size for pale stripes, determined at this eccentricity $\left(\sim 4^{\circ}\right.$ eccentricity), is about $1.5^{\circ}$ and the receptive field centers scatter over $1^{\circ}$ of visual space. This scatter should result in pale stripe coverage without gaps. These examples then support a continuous mode of representation from one pale stripe to the next.

Examples illustrating continuity from color stripe to color stripe and from disparity to disparity stripe are shown in Figures 13 and 14. Figure 13 (top) illustrates two electrode penetrations made across stripes in which electrode penetrations were placed end-to-end. Pen 7 was located entirely within a color stripe and passed through most of its width. Pen 9 crossed through the entire width of the next color stripe followed by pale/disparity/ pale stripes. As expected both of these penetrations cross roughly perpendicular to lines of isoeccentricity (Fig. 13A). Pen 7 was introduced slightly further from the V1/V2 border than the color stripe portion of Pen 9, resulting in a shift in representation closer to vertical meridian (compare locations of dashed boxes surrounding color stripe receptive field centers). Most importantly, the color stripe crossed by Pen 7 represents approximately $-3.5^{\circ}$ to $-5.0^{\circ}$ isoeccentricities, while the color stripe in Pen 9 spans -3.5 to -2.5 isoeccentricity lines (dashed boxes). There- 


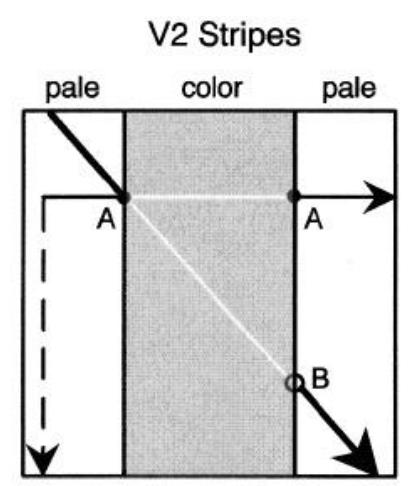

Visual Field

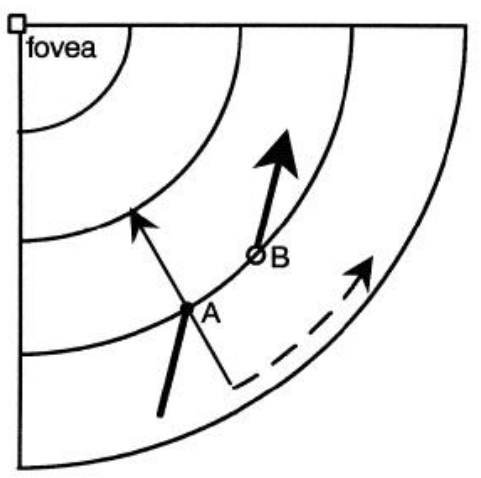

Figure 10. Schematic illustrating how penetrations skewed with respect to stripe borders result in apparent gaps in interstripe (e.g., pale to pale stripe) representation. If there is continuity from one stripe to the next like stripe, then two pale stripes separated by an intervening color stripe would share a common line of representation (more specifically, a common line of isoeccentricity). Thus, only in penetrations perfectly perpendicular to stripe borders would one see direct continuity in pale-pale stripe representation. In a skewed penetration, receptive fields recorded upon leaving the first stripe would represent a different location than that recorded at entry of the next pale stripe (i.e., there would appear to be a gap in pale stripe representation). These two points ( $A$ and $B$ ) share the same eccentricity value but different isopolar values. Thus, for skewed penetrations, one would expect continuity in the axis of changing eccentricity but not in the axis of changing polarity (see Figs. 11-14).

fore, what is represented at the end of one color stripe is again taken up at the beginning of the next color stripe. There is (Fig. $13 B$ ) approximately $0.5^{\circ}$ of overlap (between $3.0-3.5$ visual eccentricities) in coverage from one color stripe to the next.

Disparity stripes show a similar pattern. In the case shown in Figure 14 (top), two roughly orthogonal penetrations were made in V2. Pen 13, oriented almost parallel to the stripes, passed through a pale stripe and part of a disparity stripe. Pen 15 crossed through the following pale, thin, pale, and thick stripes. Continuity between the two disparity stripes is illustrated below. Disparity cell receptive fields were located at $4.0^{\circ}$ to $5.0^{\circ}$ eccentricities in Pen 13 and roughly $5.0^{\circ}$ to $6.5^{\circ}$ eccentricities in Pen 15 (Fig. 14A, dashed boxes). Even though we did not record the full width of the disparity stripe in Pen 13 , there is already a region of slight overlap in representation near the $5.0^{\circ}$ eccentricity line (Fig. 14B). Again representation appears continuous from one disparity stripe to the next.

In sum, we find evidence for rerepresentation across stripes, discontinuities at stripe borders, and continuity between like stripes. In general, receptive centers across the width of each stripe cover roughly a $1.0^{\circ}$ span of visual space, while their receptive fields cover $1.5^{\circ}-2.0^{\circ}$. Thus, there is approximately a $0.5^{\circ}$ of overlap between one stripe and the next within the same functional domain.

\section{Re-representation within single stripes}

So far, we have considered the stripe as the functional unit of representation. However, the functional clustering described previously within V2 stripes (Horton, 1984; Tootell and Hamilton, 1989; Ts'o et al., 1990b, 1991, 1994) raises the question of whether multiple maps are established even within single stripes. Some of our results support this possibility.

Figure 15 is an example in which we examined suborganization within a color stripe in V2. The optical image in Figure $15 A$ illustrates a color stripe in V2 (location indicated by hatched region in Fig. $15 B$ ) which was imaged for color versus luminance preference. Within this stripe, there is clear substructuring: alternating dark and light regions which were preferentially activated by isoluminant color and luminance stimuli, respectively. We made three tangential electrode penetrations, two across and one along, this stripe (arrows in Fig. 15B). The receptive field sequences recorded (illustrated in Fig. 15C) exhibit a clustering that is consistent with the substructure revealed by imaging. For example, in penetration $a$, following a sequence of primarily oriented broad-band cells in the adjacent pale stripe, we encountered a cluster of color disparity cells followed by a nonoriented color cell cluster. Penetration $b$ encountered a cluster of oriented red (off-response) cells followed by a cluster of primarily nonoriented green on-center red off-surround cells in the color stripe. Penetration $c$ also exhibits clustering, consisting of nonoriented color on-center cells, nonoriented color off-center cells, weakly disparity selective cells (gray bars), and then strongly disparity-selective cells (black bars).

We were interested in examining the relationship between the functional clustering within individual stripes and topographic mapping. Figure 16 illustrates the visual representation by the pale stripe cluster, the disparity cluster, and the color nonoriented cluster recorded in penetration $a$. The visual representations of these clusters show a high degree of overlap: each of these three receptive field progressions fall within a region between $1.0^{\circ}$ and $2.0^{\circ}$ azimuth and $-3.5^{\circ}$ to $-4.5^{\circ}$ elevation with a rough upward and rightward progression. As shown in Figure 17A, these progressions within each cluster are separated by discontinuities. The disparity cell cluster clearly contains a smaller degree of scatter $\left(\right.$ mean $=0.22^{\circ}$ ) than the color nonoriented cells (mean $=0.69^{\circ}$ ), a different cortical magnification factor (compare disparity slope $=0.38$ to color nonoriented slope $=1.27$ ), and is separated from the nonoriented cell cluster by a discontinuity (backward jump) at the intrastripe border. This suggests that, in addition to rerepresentation across stripes, there may also be rerepresentation within different functional domains within a stripe.

Figure 17, $B$ and $C$, illustrates functional border discontinuities for penetrations $b$ and $c$, respectively. Within the first color stripe shown in Figure 17B, there also appears to be two clusters of receptive field progressions, one (red oriented cells) at visual distance of $0.0^{\circ}-0.5^{\circ}$ and another (nonoriented color cells) around $1.3^{\circ}-1.7^{\circ}$. Figure $17 \mathrm{C}$ displays an unusual forward jump at a color/disparity border followed by a backward progression in the disparity cluster. We have seen such backward progressions associated only with disparity regions (see Fig. 19). While 

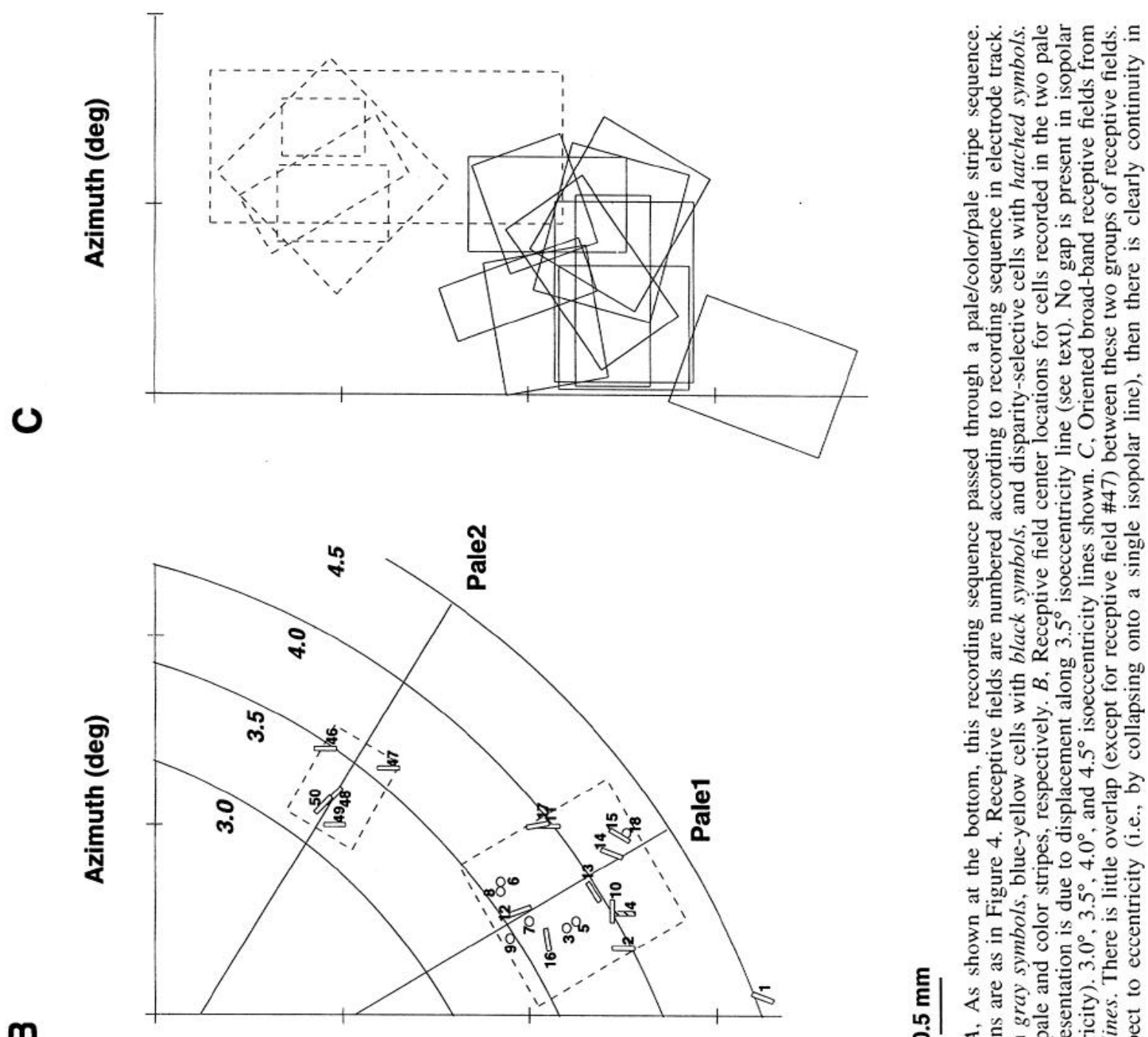

$\mathbf{m}$
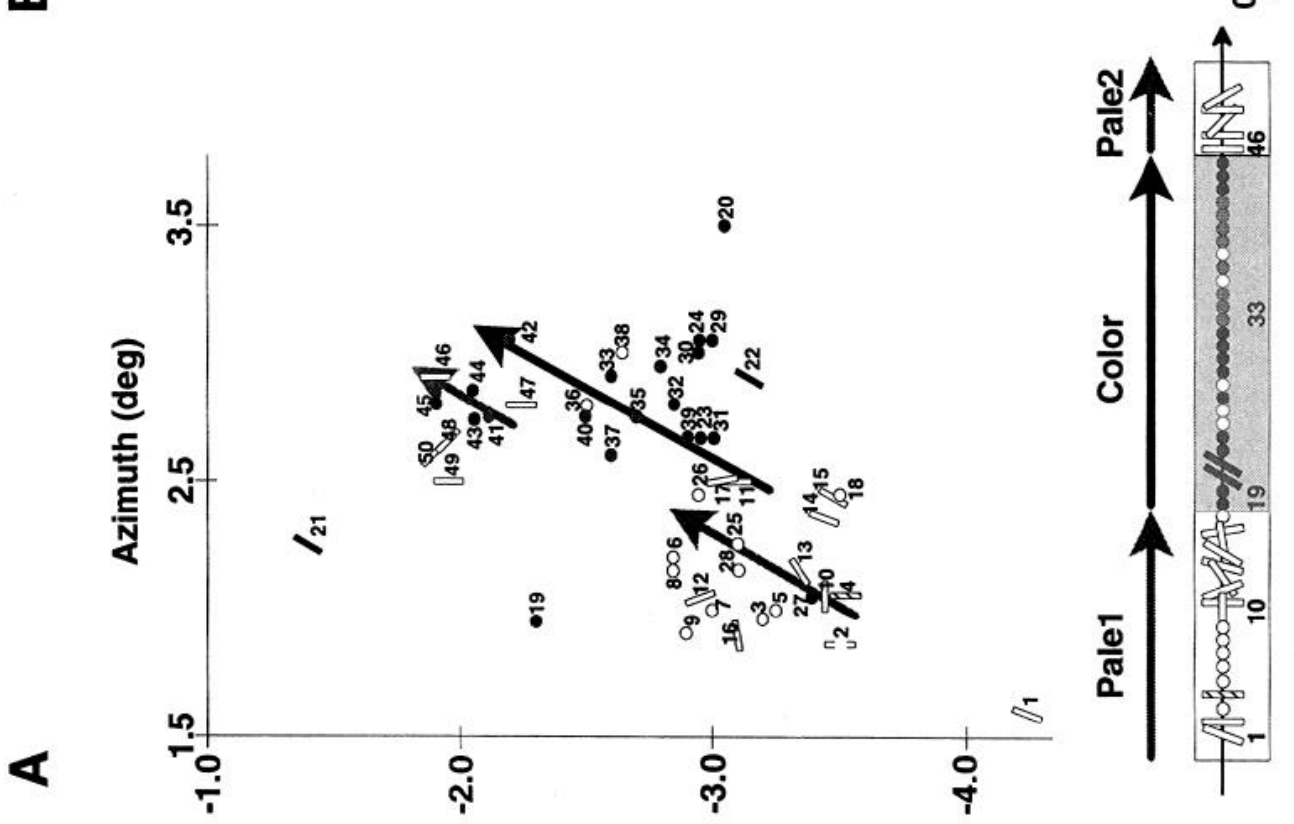

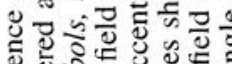

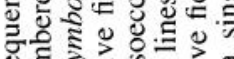

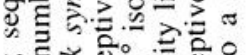

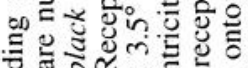

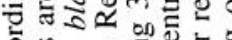

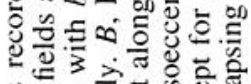

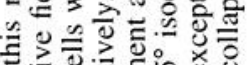
$\leq \geq 0$

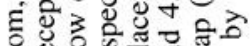

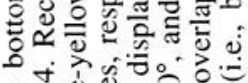
+

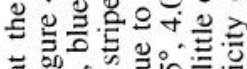

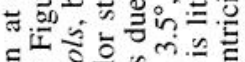

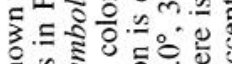

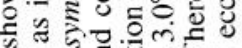

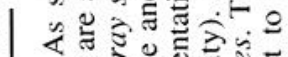

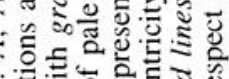

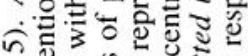

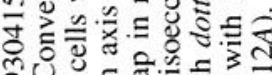

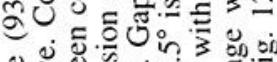

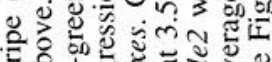

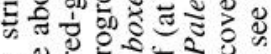

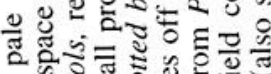
西

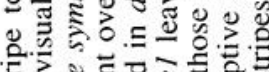
. 궐

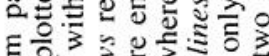
인

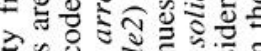

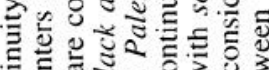
过 อำำ สิ

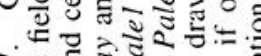
人 
A

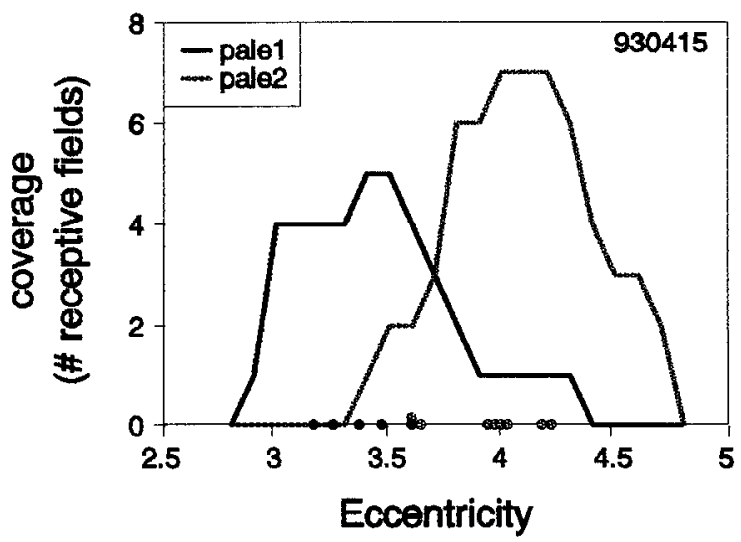

B

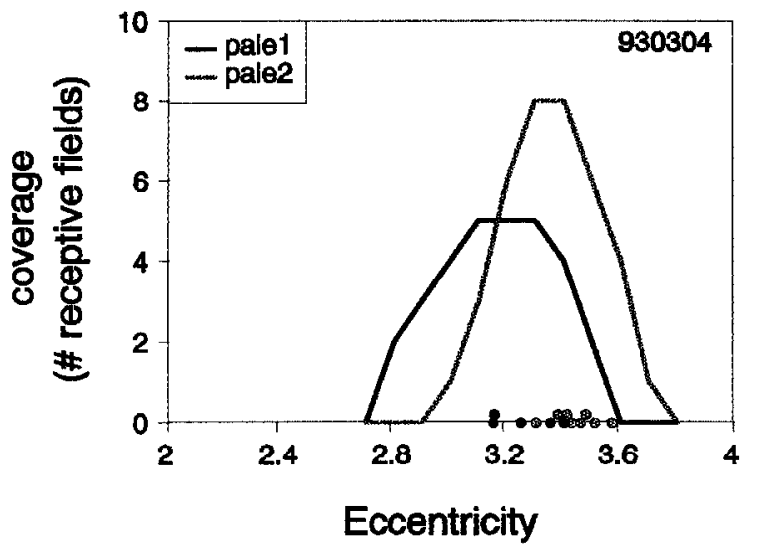

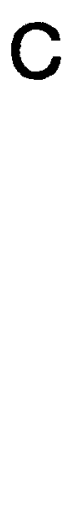

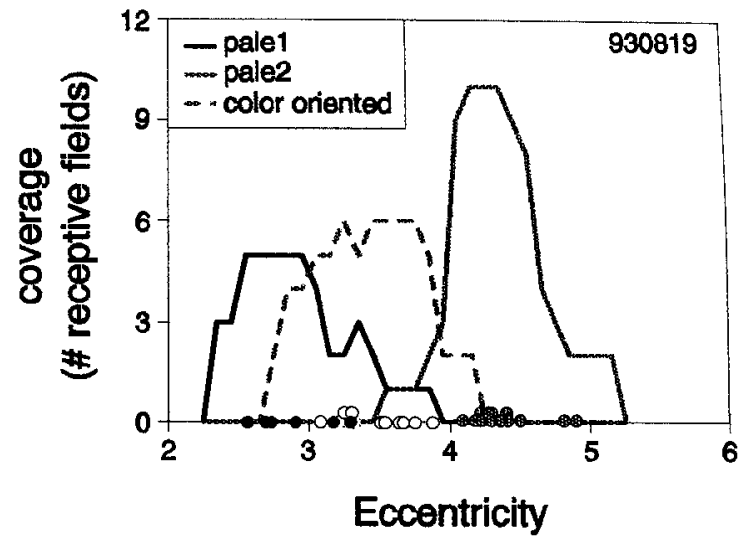

D

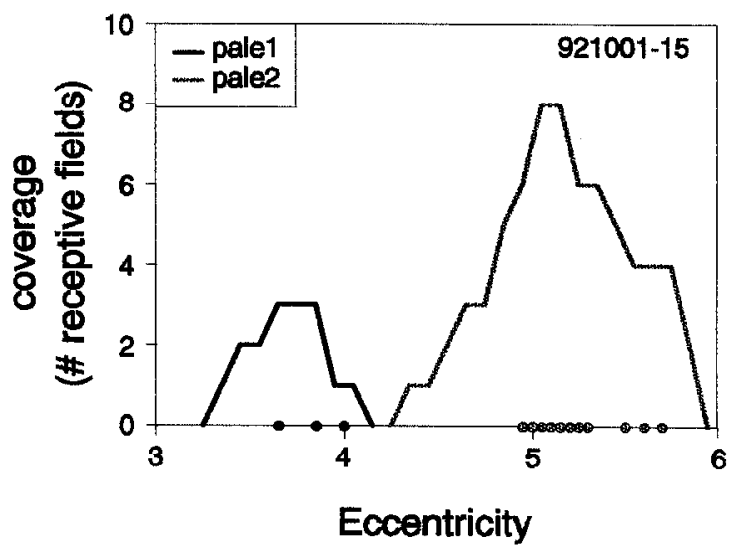

Figure 12. Coverage by pairs of successive pale stripes, as calculated only with respect to changing eccentricity. Receptive field center locations indicated by solid circles on abscissa. $A-C$ illustrate continuity between successive pale stripes. There is a small gap in continuity in $D$. $A$, Coverage of pale stripes shown in Figure 11. B, Coverage of pale stripes in penetration oriented nearly parallel to stripes. Only a portion of each pale stripe was recorded. $C$, Coverage by pale stripes in this case (shown in Fig. 4) falls to very low level around $3.5^{\circ}$ eccentricity. However, the intervening color stripe contained many color oriented cells (dotted line, open circles). This could suggest that coverage in the orientation domain may also be provided by color oriented cells. $D$, In this case (shown in Fig. 5, penetration 15), there is noticeably sparse coverage by the pale stripe system in the region around $4.2^{\circ}$ eccentricity.

we do not yet fully understand the nature of subcompartmentalization in V2 stripes, these data suggest that these functional clusters may contain independent maps.

In some cases, color stripes contain strong organization for red-green versus blue-yellow selectivity. The color stripe shown in Figure 18 (same case as in Fig. 11) contains a blue-yellow dominated region followed by a red-green region. In this case, the receptive field progressions are poorly fit by linear regression $(r=0.59)$ and no discontinuity separates the red-green/blueyellow clusters (Fig. 18A). However, the regions of visual space represented by the two clusters are highly overlapped as shown in Figure $18 B$. This overlap is again shown by coverage analysis (Fig. $18 C$ ), illustrating that each cluster covers approximately the $1.5^{\circ}-3.0^{\circ}$ region along the progression axis. Furthermore, the blue-yellow cluster (Fig. 18A) exhibits a greater degree of scatter (mean $=0.44^{\circ}$ ) than the red-green cluster (mean $=0.31^{\circ}$ ), consistent with different mapping characteristics in the blue-yellow and red-green domains. This case typifies another strategy in which visual coverage can be achieved by functional clusters via large receptive field sizes and scatter. This scheme may be adequate when receptive field size and scatter are large relative to the extent of visual field covered by the cluster.

We also see remapping in disparity clusters. In the example shown in Figure $19 B$ (top), the recording sequence included a long sequence of disparity cells, half of which were broad-band disparity cells and half primarily disparity cells selective for red stimuli. The receptive fields of these two disparity sequences (Fig. 19A, light lines vs bold lines) are highly overlapped (extent indicated by light bar and bold bar at left). In addition, there is a clear discontinuity in progression at the broad-band disparity and red disparity border (Fig. 19B, arrow). Note that similar to the disparity cell progression in Figure $17 \mathrm{C}$, both of these disparity sequences map as a backward progression (negative slope of the regression lines). Thus, at the color/disparity border there is an actual reversal in progression, followed by a jump forward at the intradisparity stripe border, followed by another reversal at the disparity/pale stripe border. We have observed reversed progressions only in association with disparity clusters.

\section{Mapping parameters within different functional stripes: scatter, receptive field size, and cortical magnification}

Scatter with respect to depth. For each of the color, pale, and disparity stripes, we have found no consistent relationship between scatter (normalized for eccentricity) and depth (with respect to the cortical surface); nor with scatter and eccentricity, nor receptive field size and depth, nor receptive field size and 

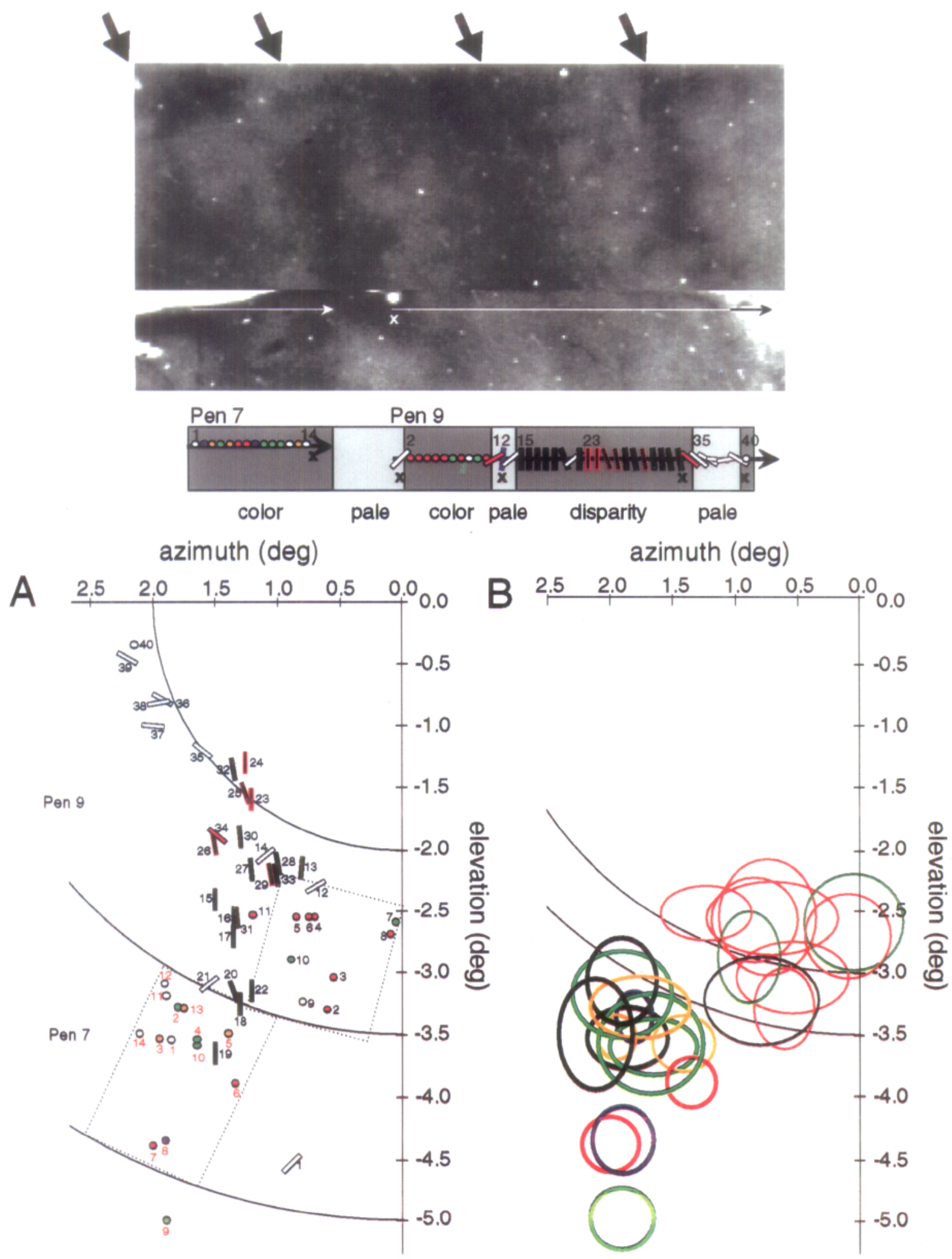

Figure 13. Continuity in representation from color stripe to color stripe (930405 Pen 7 and Pen 9). Top, Location of Pen 7 and Pen 9 relative to stripes. Lesions (marked by $X$ ) were made at the end of Pen 7 , the beginning of Pen 9 , the blue oriented cell in the first pale stripe in Pen 9 , the end of disparity sequence in Pen 9, and the end of Pen 9. One of these lesions is seen in the cytochrome oxidase stained section shown above (white $x$ ). Position of Pen 7 and Pen 9 are indicated on tissue by white arrows. Interruption in section is due to cutting cortical tissue into two parts, surface V2 and V2 on posterior bank of lunate, for ease of histological processing. Darkly staining stripes are indicated by black arrows at top. A, Receptive field center locations of all cells recorded in Pen 7 and Pen 9. Dashed boxes indicate visual regions represented by color stripes in Pen 7 and Pen 9. Shift in isopolarity is due to the fact that Pen 7 was located further from the V1/V2 border than the color stripe portion of Pen 9. Notice continuity in eccentricity representation from Pen 7 to Pen $9 . B$, Receptive field plots of cells recorded in color stripes. Color receptive fields recorded in Pen 7 (thick line circles) overlap in eccentricity $\left(\sim 3.0^{\circ}-3.5^{\circ}\right)$ with those recorded in the color stripe in Pen 9 (thin line circles). 


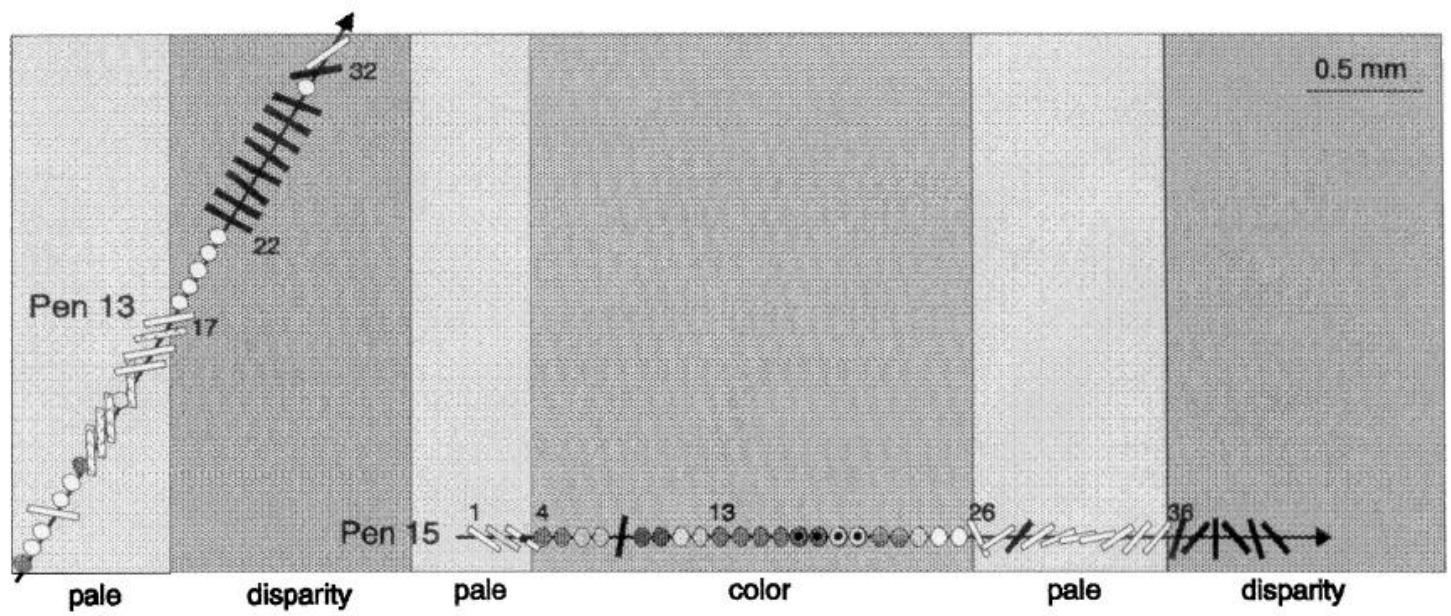

A

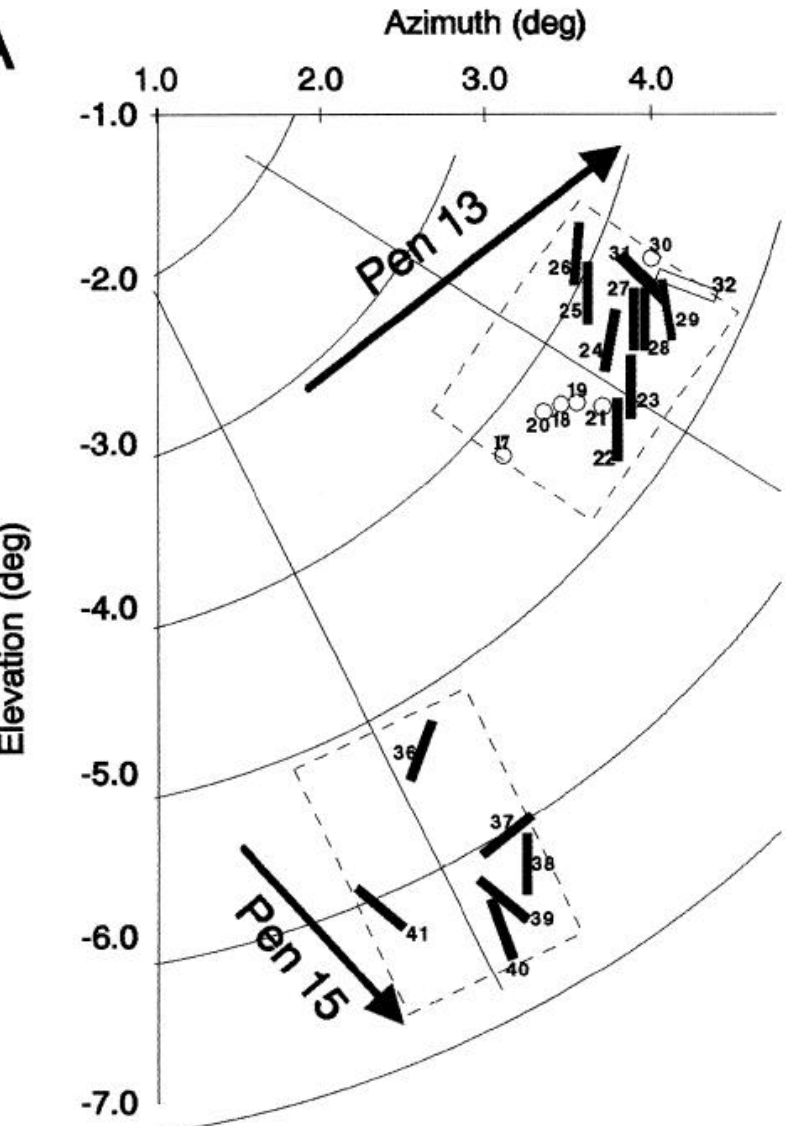

B

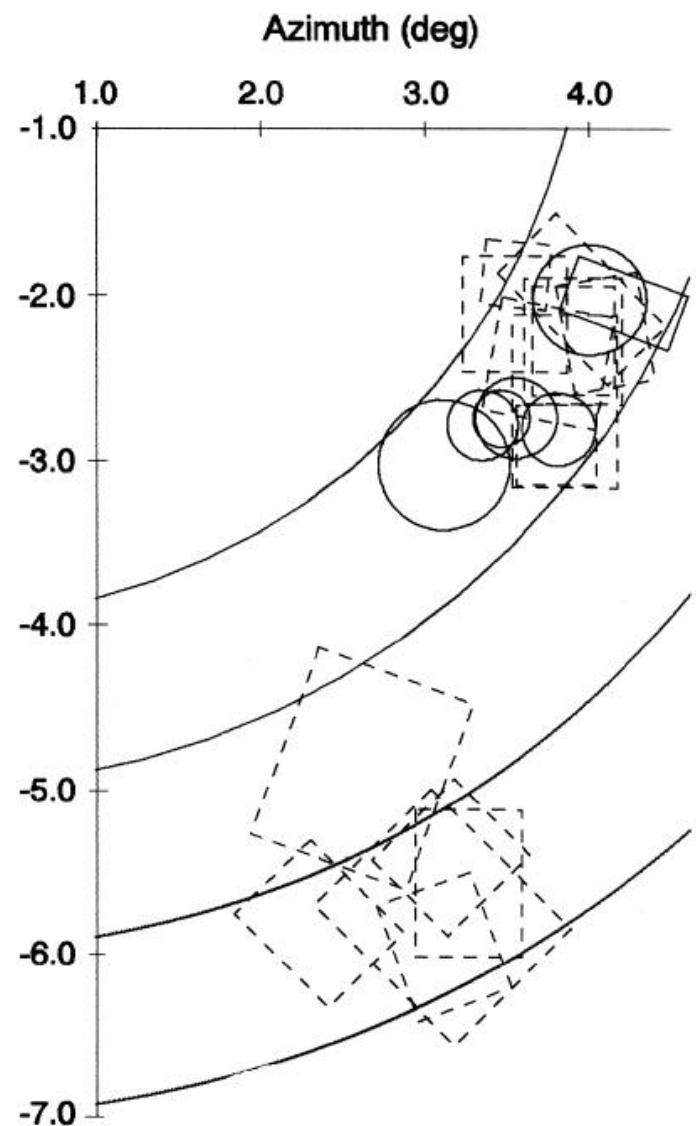

Figure 14. Disparity to disparity stripe continuity (same case shown in Fig. 5). Top, Two successive disparity stripes were recorded in Pen 13, oriented nearly parallel to the stripes, and Pen 15, oriented across stripes. Continuity between the two disparity stripes is illustrated below. $A$ Dashed boxes are drawn around disparity receptive field centers. Pen 13 disparity cell receptive fields were located at $4.0^{\circ}$ to $5.0^{\circ}$ eccentricities and roughly $5.0^{\circ}$ to $6.5^{\circ}$ eccentricities in Pen 15 . B , Receptive fields of cells shown in $A$. Conventions are as in Figure $6 A$. Coverage of Pen 13 falls roughly between 4.0 and 5.0 eccentricities; that of Pen 15 between 5.0 and 7.0 eccentricities. Even though we did not record the full width of the disparity stripe in Pen 13, there is already a region of slight overlap in representation near the $5.0^{\circ}$ eccentricity line.

eccentricity. We have also observed no significant relationship between end-inhibition and either receptive field size or length, or cortical depth.

Comparisons between pale and color stripes. We compared the degree of visual scatter in the color, pale, and disparity stripes. As a measure of scatter, we used the visual distance between the receptive field centers of two successive units re- corded within a stripe normalized by the average visual eccentricity between those units. In general, scatter between successive units recorded at $2^{\circ}-5^{\circ}$ eccentricity averaged between $0.25^{\circ}$ and $0.5^{\circ}$ (pale mean $=0.3^{\circ}$, color mean $=0.39$, disparity mean $=0.31$ ). For color stripes, we additionally made separate scatter calculations for color oriented and color nonoriented cells. Since the magnitude of visual scatter recorded may be affected by the 

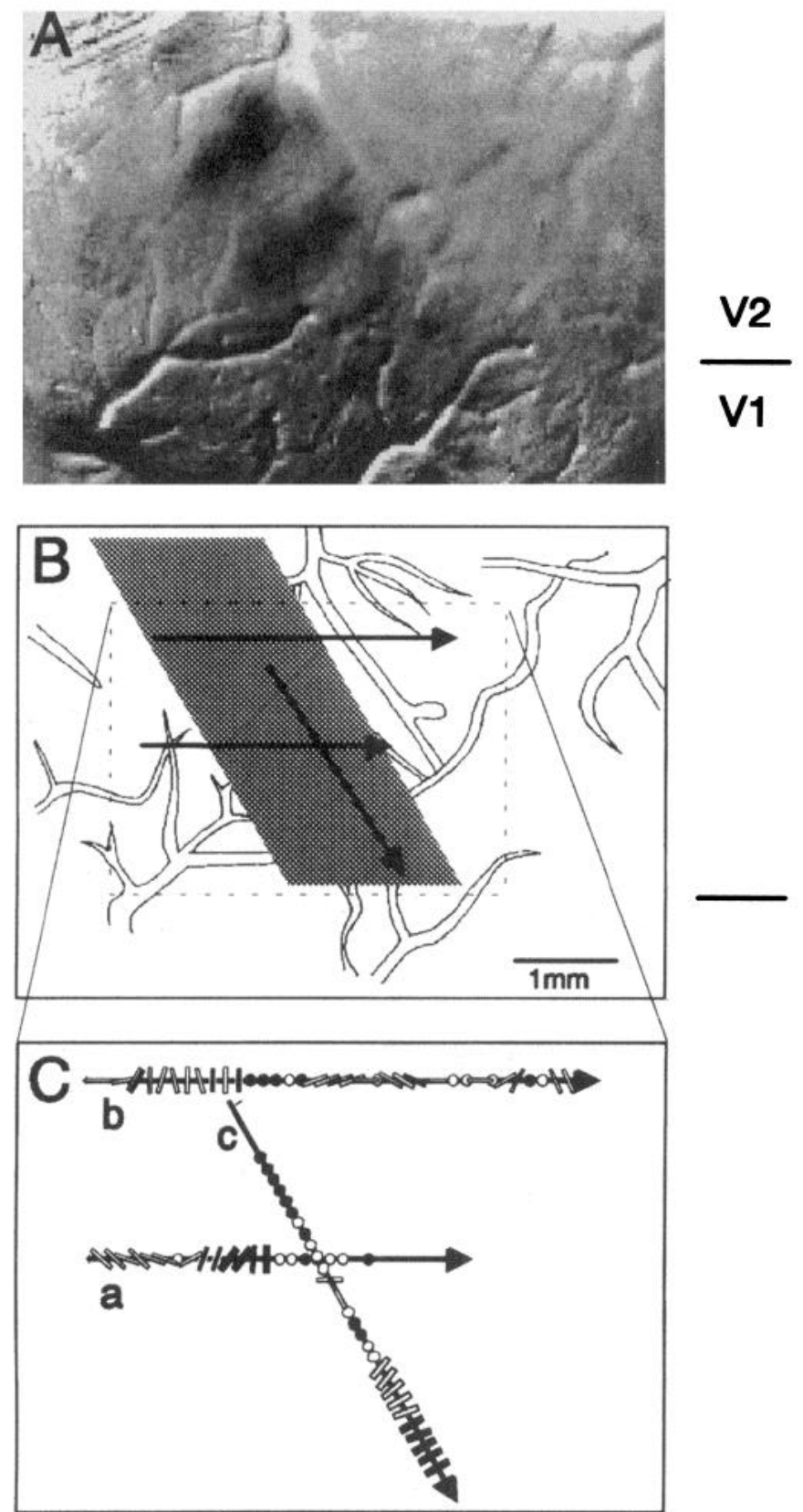

Figure 15. Substructure within a color stripe. A, Optical image of color stripe reveals substructure when imaged for color (black regions) versus luminance (white regions). Location of V1/V2 border indicated at right. $B$, Illustration of cortical region shown in $A$. Vasculature are drawn. Position of color stripe (hatched region) and three tangential electrode penetrations (two across and one along this stripe) are shown. Region in dashed box is shown expanded in $C$. Scale bar, $1 \mathrm{~mm}$ (applies to $A$ and $B$ ). $C$, Receptive field types are indicated by symbols as in Figure $3 C$. There is evidence of functional clustering along each of penetrations $a, b$, and $c$ (see text). For color version, see Figures 16 and 17 .

direction of the electrode penetration in cortex (see Anisotropy of V2 map), we made comparisons of scatter between stripes recorded within the same penetration.

We find some differences in scatter between pale and color stripes (see Fig. 20). Out of 13 intrapenetration pale/color comparisons (from nine penetrations which contained adjacent pale and color stripes), pale stripe scatter was significantly smaller
(Student's $t$ test, $p<0.1)$ in six and either not different $(n=5$, $p>0.1)$ or slightly greater $(n=2, p<0.1)$ in seven. We then considered the position of these pale stripes relative to color stripes. In almost all cases, we found that pale stripes directly medial to color stripes were those with smaller scatter and those directly lateral to color stripes had equal or greater degrees of scatter. This difference in pale stripe scatter is unlikely be attributed to eccentricity changes since scatter measures were normalized for eccentricity. Nor is it related to cortical depth since some penetrations crossed increasing and some decreasing eccentricities.

Differences in receptive field size paralleled differences in scatter. [Receptive field sizes of V2 cells typically ranged from $0.25^{\circ}$ to $0.75^{\circ}$ and sometimes reached up to $2.0^{\circ}$. Receptive field size for all broad-band oriented cells recorded averaged 0.57 , for color cells 0.63 , and for disparity cells $0.60^{\circ}$.] Five out of six pale stripes medial to color stripes demonstrated smaller (or equal) receptive field sizes, whereas seven out of seven pale stripes lateral to color stripes demonstrated equal (or larger) receptive field sizes. In general, receptive field sizes of color cells were larger than those of disparity cells. One possible interpretation of these differences in scatter and receptive field size is that two types of pale stripes exist in V2-one more closely associated with color stripes and perhaps one with disparity stripes. This notion is further supported by comparisons with disparity stripes.

Comparisons with disparity stripes. We recorded from three penetrations which crossed both disparity and pale stripes. There was no significant difference in scatter or receptive field size in any of these pale/disparity comparisons (Fig. 20). Of the three penetrations which crossed both disparity and color stripes, two had greater scatter in color stripes than disparity stripes. The third, which contained a cluster of broad-band disparity and a cluster of red-selective disparity cells (see Fig. 19), had greater scatter in the disparity stripe. However, this increase scatter is due solely to the red-selective disparity cluster within the stripe. Even within the disparity stripe the scatter within the red-selective disparity cluster was significantly greater $(p<0.001)$ than that in the broad-band disparity cluster.

Color-oriented cells. We considered the possibility that color stripes have greater scatter than pale stripes because they contain more diverse cell types. To address this, we made separate oriented and nonoriented color comparisons. Pale stripes have consistently less scatter than nonoriented cells in color stripes (10 out of 10 comparisons). Of six pale/color-oriented comparisons, two were significantly different and four were not. Our impression is that color oriented cells come in two species. Those of the color stripe variety are usually interspersed among nonoriented color cells, while those associated with pale stripes are often clustered near pale/color stripe borders. Of four penetrations which contained significant numbers of both color oriented and broad-band oriented cells, color-oriented clusters in pale stripes did not differ from broad-band oriented cells in pale stripes either in terms of scatter or receptive field size. They did differ, however, from color stripe nonoriented cells in scatter (two penetrations: $p<0.1, p<0.01$ ) and receptive field size (two penetrations: $p<0.01, p<0.1$ ). In contrast, color oriented cells interspersed within color stripes display scatter and receptive field size similar to nonoriented color cells, but not to broadband oriented cells (scatter: $p<0.01, p<0.05$; receptive field size: $p<0.1, p<0.1)$.

Cortical magnification. We calculated cortical magnification 
factors for each stripe. We considered only those stripes across which we were confident we had recorded the full width. We did not attempt to consider stripe subcompartments for these calculations and simply took the cortical distance across the entire stripe (determined from the number of units recorded corrected by the electrode angle to the surface) and divided by the visual distance between the most extreme receptive field centers along the axis of progression. The magnification factors obtained are plotted in Figure 21. Pale stripe $(n=6)$, color stripe $(n=$ $6)$, and disparity stripe $(n=2)$ magnification factors are 1.44 $\mathrm{mm} /$ degree, $1.40 \mathrm{~mm} /$ degree, and $1.25 \mathrm{~mm} /$ degree, respectively. Cortical magnification factors derived from inverse-slopes of the receptive field progression plots (such as those slowni in Figs. $8,17,19)$ are also consistent with Figure 21. These are 1.32 $\mathrm{mm} /$ degree \pm 0.23 for pale stripes, $1.53 \mathrm{~mm} /$ degree \pm 0.54 for color stripes, and $1.48 \mathrm{~mm} /$ degree \pm 0.25 for disparity stripes. Thus, by both measures the cortical magnifications for the three stripe systems in V2 are roughly the same.

As mentioned previously, there is considerable variability in V2 stripes. One such example has been shown in Figures 13 and 19 in which there are two color stripes with no intervening disparity stripe between them. Interestingly, in this case the cortical magnification factor for the following disparity stripe (Fig. $19)$ is significantly lower $(0.83 \mathrm{~mm} /$ degree $)$, on the order of half the magnification of most V2 stripes. It is possible that in order to compensate for the missing disparity stripe, this disparity stripe had to cover twice as much visual field, resulting in the roughly halved magnification factor.

\section{Anisotropy of V2 map: along versus across the stripes}

Given the difference in functional organization in the two dimensions in V2-that is, across stripes and along stripes-it was interesting to examine whether certain features of V2 maps (such as scatter and magnification) exhibit anisotropic tendencies. In this section we consider this issue with respect to receptive field scatter and cortical magnification.

Scatter. We compared the degree of scatter along vs. across the stripes in V2 by calculating the normalized scatter (see Materials and Methods) for each of the stripes in the two orthogonal penetrations illustrated in Figure 5. Scatter in color stripes is significantly less (Student's $t$ test $p<0.0003$ ) along (mean $=$ $0.23^{\circ}$, normalized mean $=0.06^{\circ}$ ) than across (mean $=0.52^{\circ}$, normalized mean $=0.11^{\circ}$ ) the stripe. In pale stripes and disparity stripes, the average degree of scatter along the stripe was less than that across stripes; however, these differences did not reach statistical significance.

Cortical magnification. To more accurately compare cortical magnification factors along versus across stripes, we felt it was important to make comparisons within the same animal and preferably within the same stripes. We therefore made the calculations illustrated in Figure 22. Figure $22 A$ illustrates two parallel electrode penetrations which were made exactly $1 \mathrm{~mm}$ apart (as determined by the electrode micromanipulator markings) on the cortical surface across a pale and a color stripe in V2. We first chose a group of cells in each stripe and then chose two cells within the same stripe roughly equidistant from the circled group of cells, one $1 \mathrm{~mm}$ distant along the stripe and a second $1 \mathrm{~mm}$ away across the same stripe. We then calculated several inverse cortical magnification factors between the circled cells and the single units approximately $1 \mathrm{~mm}$ away. The inverse magnification factors proved to be quite different along versus across the stripes. The calculations across the pale stripe produced numbers falling in the range of 0.7 to 1.0 range (Fig. $22 B$, white squares), while the along calculations resulted in factors ranging from 1.5 $-2.0 \% \mathrm{~mm}$ (Fig. $22 \mathrm{~B}$, white circles). The same calculation done between a group of color cells in the color stripe and a second color cell, either across the same stripe (red squares) or along the same stripe (red circles), produced similar results.

Inverse magnification factors along the stripes are thus significantly higher than those across stripes. Notice that cortical magnification factors across stripes calculated from these numbers (by inversion) fall in the same range $(0.5-0.7 \mathrm{~mm} /$ degree) as those calculated previously. Thus, consistent with the presence of multiple representation in V2, cortical representation across the stripes is two to three times as extensive in the axis across the stripes than along.

\section{Discussion}

\section{Summary}

The findings reported in this article support a discontinuous model of visual representation (see Fig. 1). This mapping can be described as a multiple and discontinuous representation of visual space across the three types of functional stripes in V2. Each locus of visual space is represented (at least) three timesonce in the color domain, once in the orientation domain, and once in the disparity domain. This mode of representation is characterized by continuity within stripes, discontinuities at stripe borders, and by continuity from one stripe to the next like stripe. This triple representation is also accompanied by stripespecific mapping parameters such as different receptive field sizes, scatters, and cortical magnification factors associated with each stripe type. In addition, we have obtained some evidence for remappings within single stripes and believe that these are closely associated with functional modules previously described within V2 stripes. These findings raise several issues about cortical topographies which we address below.

\section{Multiple maps within single cortical areas: a common strategy for representing multiple functional domains}

Precedence for multiple representation within cortical areas can be found other studies. A re-representation of visual space across different functional domains was initially suggested by Hubel, Wiesel, and LeVay (1974) with respect to ocular dominance columns in V1 (also see Hubel and Wiesel, 1977; Blasdel and Fitzpatrick, 1984). They schematically depicted the discontinuous mapping of left eye and right eye progressions recorded in a tangential penetration through layer $4 \mathrm{C}$ in V1. Within each ocular dominance column, there is a continuous visual progression covering an equal amount of visual field. However, there is a shift in visual representation between the left and right eye domains such that at the left eye/right eye border the visual fields jump back half the distance of the previous progression. They thus termed this a "two steps forward, one step back" progression. In V2, we also find a similar discontinuous and shifted pattern of representation. Other reports of visual representation in V2 have suggested this repeated representation. Rosa et al. (1988) predicted a re-representation across functional domains to explain data obtained with multiple vertical electrode penetrations in Cebus monkey V2. Zeki and Shipp (1987) and Zeki (1990) also briefly reported findings consistent with those reported here. While they did not classify cells on the basis of disparity tuning, they reported on the basis of three long tangential penetrations across stripes in V2 greater overlap in the receptive fields recorded within adjacent stripes (e.g., a thin and 
Figure 16
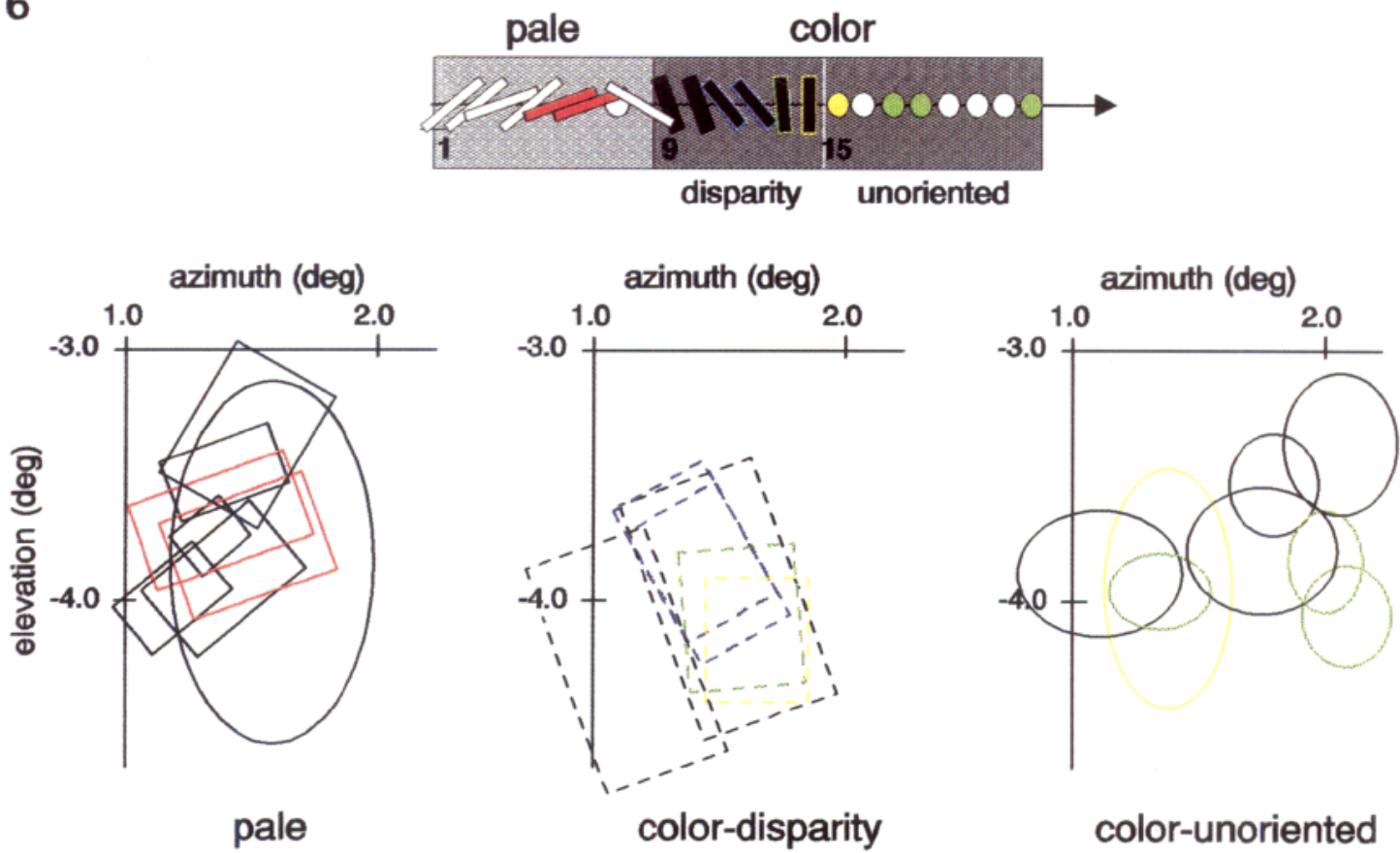

color-disparity

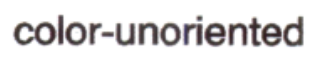

\section{Figure 17}
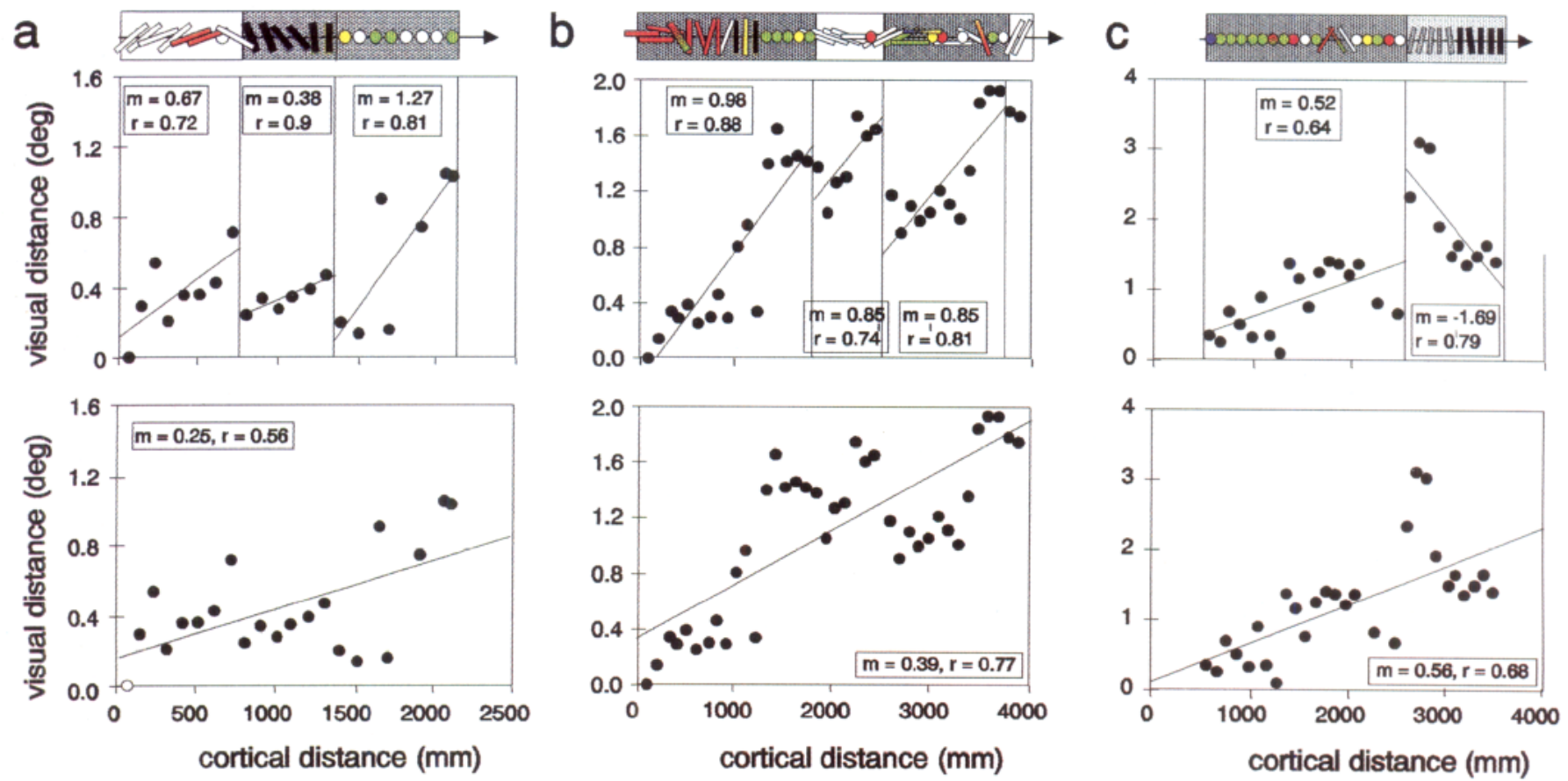

Figure 16. Re-representation by stripe subcompartments. Receptive fields recorded in pale stripe, color disparity substripe, and color nonoriented substripe regions (top) are plotted in $A-C$, respectively. Each of these three clusters represent approximately the same region of visual space (between $1.0^{\circ}$ and $2.0^{\circ}$ azimuth and $-3.5^{\circ}$ and $-4.5^{\circ}$ elevation). Thus, not only is there representation across functional stripes but also within different functional domains within a stripe.

Figure 17. Receptive field progression graphs for penetrations $a-c$ shown in Figure 15. All distances measured relative to first cell in each penetration. Overall regressions shown below. Stripe (or cluster) specific regressions shown above (slopes, $m$, and regression coefficients, $r$, shown), Penetration $a$, Discontinuities can be seen not only at the pale/color border, but also within the color stripe between the disparity and the nonoriented color cell clusters. The disparity cluster also exhibits a smaller degree of scatter than the other two clusters. Penetration $b$, Color/pale/color sequence. Some clustering (red oriented cluster and green nonoriented cluster) is seen within the first color stripe. Penetration $c$, Substructure along color stripe. There is a clear discontinuity (a jump forward) between the color nonoriented cluster and the disparity cluster. 
A

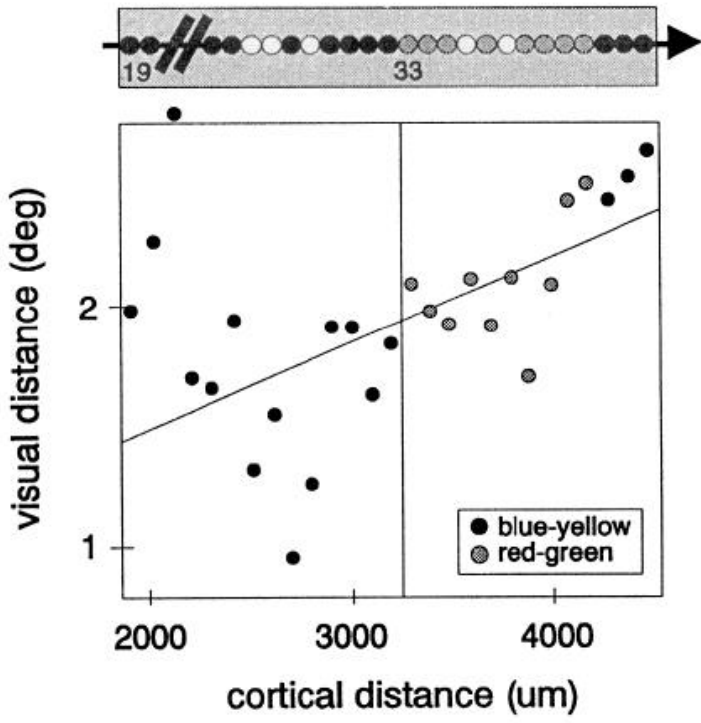

B

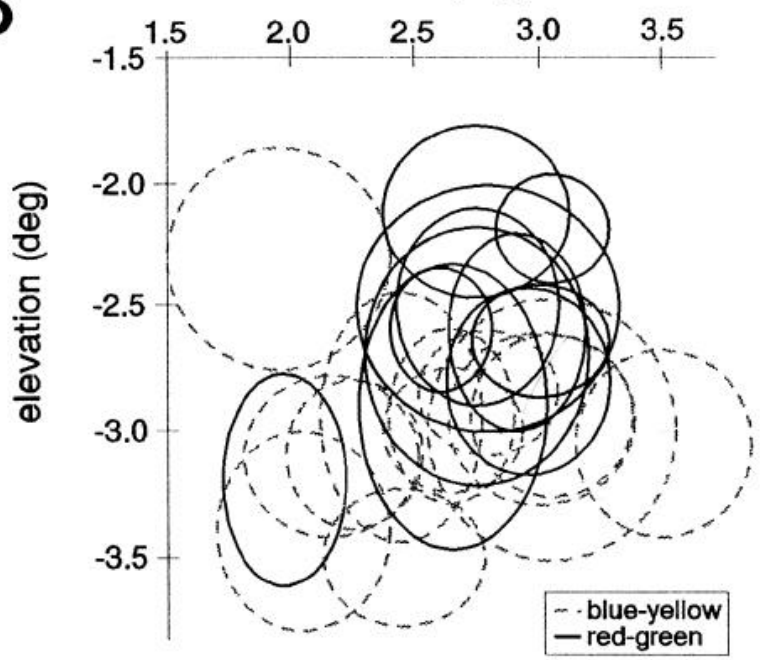

C

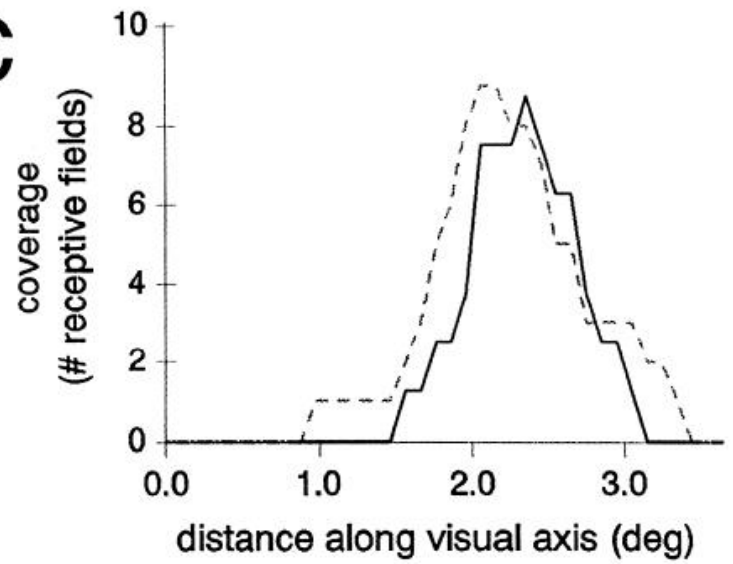

Figure 18. A, A tangential penetration which recorded a blue-yellow cluster (black symbols) followed by a primarily red-green cluster (gray symbols) in a V2 color stripe (same case shown in Fig. 11). Receptive field progression plot is shown below. All distances measured from cell a pale stripe) and little overlap in those recorded from two functionally similar stripes. Discontinuities at or near functional borders similar to those described in this article were also seen.

In area MT, anatomical and functional studies (Tootell et al., 1985; Krubitzer and Kaas, 1990a,b) suggest the presence of two interdigitated organizations, which may be involved in the processing of local versus global motion cues (Born and Tootell, 1992). Given such functional and connectional distinctions between these two compartments, it is possible that MT may contain multiple visual maps as well (cf. Gattass and Gross, 1981; Albright and Desimone, 1987). In nonvisual areas, instances of re-representation can also be found, although none of these display as systematic remapping as in primate V2. For example, using multiple vertical electrode penetrations, Sur et al. (1981) studied the distribution of rapidly adapting and slowly adapting cutaneous neurons in the digit representation of primary somatosensory cortex of owl and macaque monkeys. Their study suggested that each modality is represented in irregular wavy bands which are interweaved in a complex fashion, suggesting that the skin of each digit is represented twice in area $3 \mathrm{~b}$. A similar finding was reported for cat somatosensory cortex (Sretevan and Dykes, 1983). Thus, repeated topographic representation is a strategy commonly used by cortical areas that must incorporate multiple functional domains within the two-dimensional structure of neocortex.

Comparison of color, pale, and disparity stripes in V2: receptive field size, scatter, and cortical magnification

Given that V2 is divided into three topographic representations, we would like to know how similar these maps are. This question can be addressed by comparing mapping parameters such as receptive field sizes, scatter, and cortical magnification.

Relative receptive field size and scatter in different stripes. We find greater receptive field size and scatter for the color stripes than the disparity stripes. Although at this point it is difficult to relate these findings to psychophysical measures of color, form, and disparity perception, it can be argued that the color system would require a less precise mapping than either the form or disparity system. The visual system's ability to accurately localize isoluminant color borders is far less precise than its ability to localize luminance edges or disparity boundaries (cf. Morgan and Aiba, 1985). It is possible that the nonoriented color system serves to fill in the regions bounded by luminance or disparity contours and would require less positional precision. An alternative possibility is that color stripes contain more diverse cell types and so exhibit greater apparent scatter. However, scatter in color patches dominated by a single color opponency is still greater than disparity and pale stripes, sug-

$\leftarrow$

$\# 1$. Regression fit is poor $(m=0.00037, r=0.59)$ and no discontinuity occurs at border between the two color clusters. In this case coverage is achieved via large receptive field size and scatter, as shown in $B$ and $C$. The receptive field of cell $\# 21$, indicated by dot above graph, was located $2.97^{\circ}$ from cell \#1 and was not included in the regression calculation. B, Receptive field plots of nonoriented blue-yellow (gray dotted circles) and red-green (black circles) cells recorded show high overlap. [The three blue-yellow cells at the end of the color recording sequence (cells 43-45; see part $A$ ) are not drawn since we cannot consider them part of either the blue-yellow cluster (cells 19-32) or the redgreen cluster (cells 33-42).] $C$, Receptive field coverages by the blueyellow (gray dotted line) and red-green (black solid line) clusters (shown in part $B$ ) are highly overlapped. 


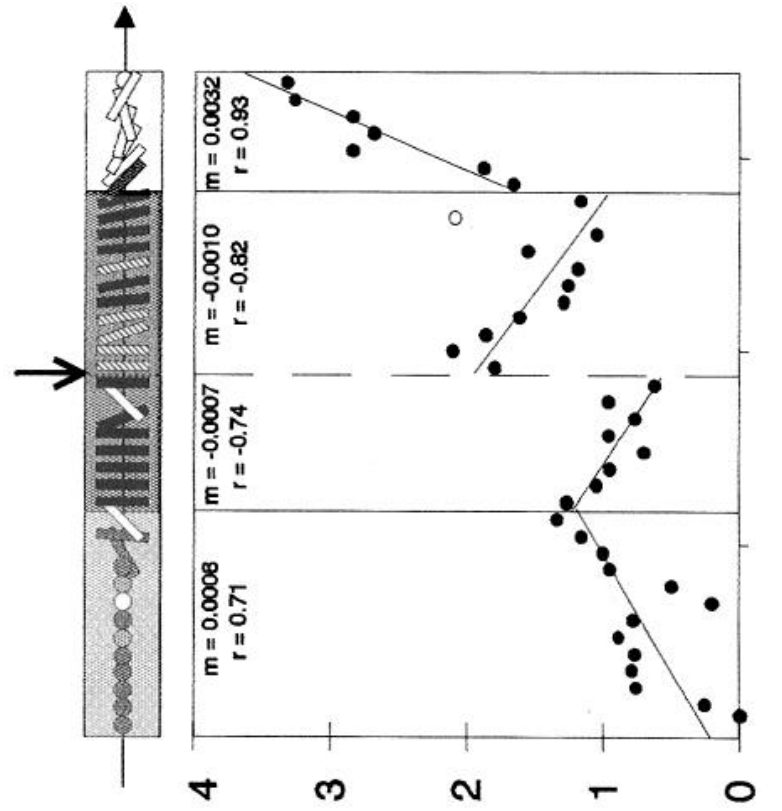

$m$

(6өp) өэuełs!p jens!n

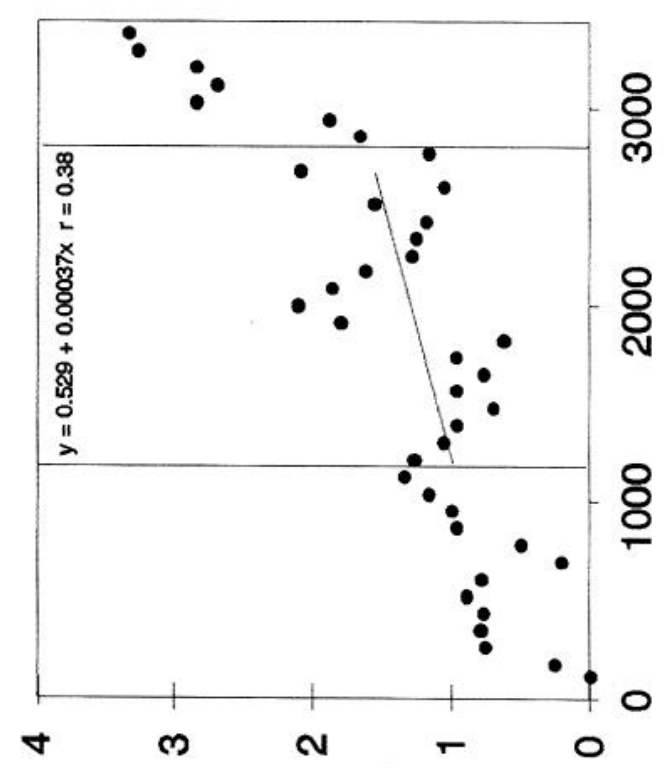

(6өр) өэuełs!̣ jens!̣

elevation (deg)

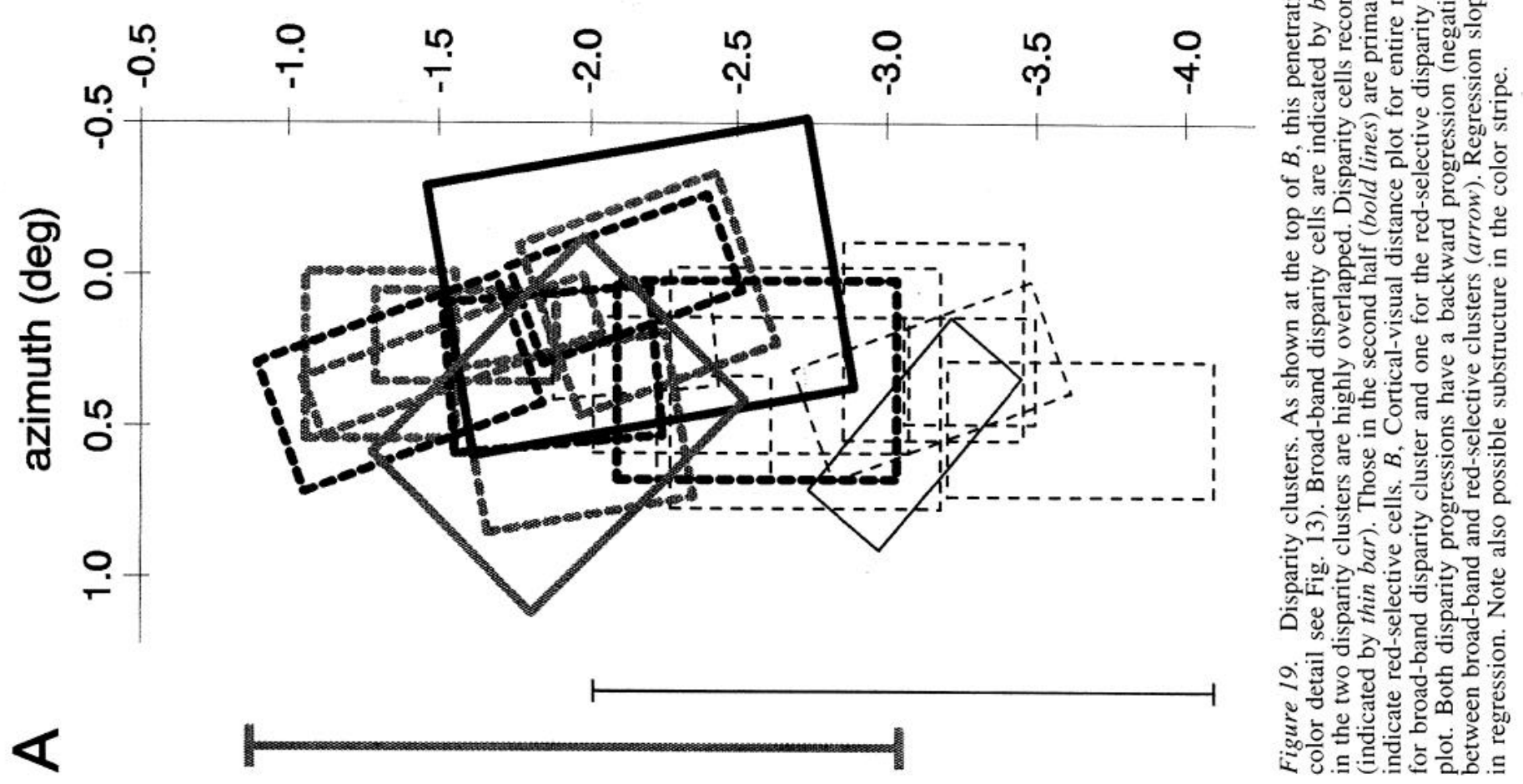



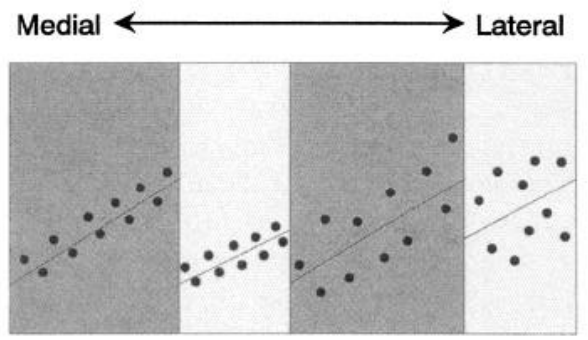

\section{SCATTER}

$$
\text { disparity } \underset{(3 / 3)}{=} \text { pale } \underset{(6 / 6)}{<} \text { color } \underset{\substack{(5 / 7) \\(2 / 7)}}{=} \text { pale }
$$

RF SIZE

$$
\text { disparity } \underset{(3 / 3)}{=} \text { pale } \underset{\substack{(3 / 6)\\
}}{<} \text { color } \underset{\substack{(6 / 3) \\
(2 / 3)}}{=} \begin{gathered}
< \\
\end{gathered}
$$

Figure 20. Evidence for two types of pales stripes: comparison of receptive field sizes and scatter in V2 stripes recorded within single electrode penetrations. In general, color stripes contained greater scatter than disparity stripes (see text). Pale stripes with scatter smaller than that in color stripes were located medial to color stripes (six out of six comparisons) and had similar scatter to the adjacent disparity stripe (three out of three comparisons). In contrast, pale stripes lateral to color stripes had scatter equal to (five out of seven) or greater than (two out of seven) the adjacent color stripe. Pale stripe cells also tended to have different receptive field sizes depending on stripe location. All pale stripes with smaller receptive field sizes (three out of six comparisons) were located medial to color stripes and had receptive field sizes similar to the adjacent disparity stripe (three out of three). Most pale stripes (7 out of 10) with receptive field sizes larger or equal in size to color stripes were located lateral to color stripes. These differences in receptive field size and scatter support a dichotomy of pale stripes in V2: those more closely associated with disparity stripes (located medial to color stripes) and those more closely associated with color stripes (located lateral to color stripes).

gesting that cell type diversity is not the primary contributor to increased scatter in color stripes.

One fundamental question about V2 stripe organization is why there are twice as many pale stripes as either color or disparity stripes. While such an arrangement may be a byproduct of developmental events, it appears that there may indeed be some functional differences within the pale stripe population. Our scatter analysis has unexpectedly revealed that there may be two types of pale stripes in V2 one with larger scatter similar to that of color stripes (consistently located lateral to color stripes) and one with smaller scatter similar to that of disparity stripes (consistently located medial to color stripes). We are currently examining the possibility that alternating pale stripes in $\mathrm{V} 2$ have differential interareal as well as intra-areal connectivities.

Relative cortical magnification in different stripes. The cortical magnification factors calculated in this article across stripes at an eccentricity of $2-5^{\circ}$ are roughly $1.3 \mathrm{~mm} /$ degree for each of the three stripe systems. One would expect these figures to be on average one third that reported for overall cortical magnification at similar eccentricity. That is, at this eccentricity a full cycle of representation would cover on average roughly 4 $\mathrm{mm}(=1.3+1.3+1.3)$ of cortex per degree of visual representation. The magnification factors reported by Gattass et al. (1981) at a comparable eccentricity displays a considerable degree of scatter and is somewhat lower than the overall magnification factor predicted by this study (at $3^{\circ}-5^{\circ}$ eccentricity, range $=0.2-2.4 \mathrm{~mm} /$ degree; Gattass et al., 1981). However,

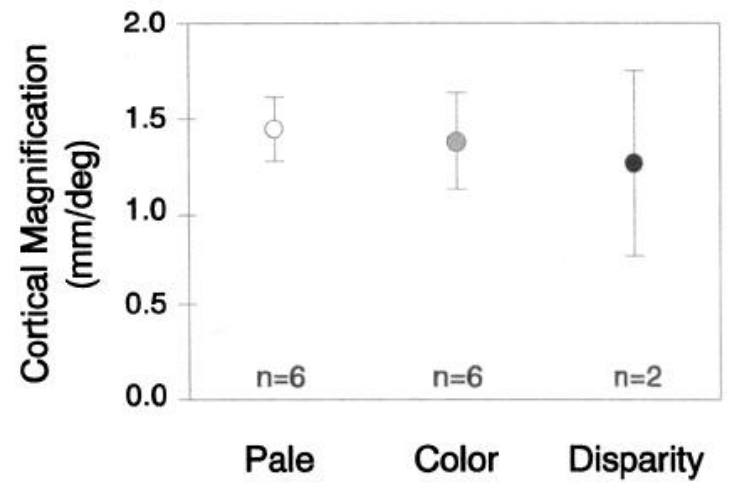

Figure 21. Cortical magnification factors in pale $(n=6)$, color $(n=$ $6)$, and disparity $(n=2)$ stripes. Plotted are cortical magnification values calculated by dividing the width of the stripe by the visual distance between the most extreme receptive field centers along the axis of progression. Cortical magnification factors derived from inverse-slopes of the receptive field progression plots are similar: $1.32 \mathrm{~mm} /$ degree \pm 0.23 for pale stripes, $1.53 \mathrm{~mm} /$ degree \pm 0.54 for color stripes, and $1.48 \mathrm{~mm} /$ degree \pm 0.25 for disparity stripes. Thus, by both measures the cortical magnifications for the three stripe systems in V2 are roughly the same.

given the significant differences in spatial sampling (every 100 $\mu \mathrm{m}$ in this study and every 1-2 $\mathrm{mm}$ in Gattass et al., 1981), lack of stripe localization in the Gattass study, and the differences in estimation of cortical distance (distance along electrode

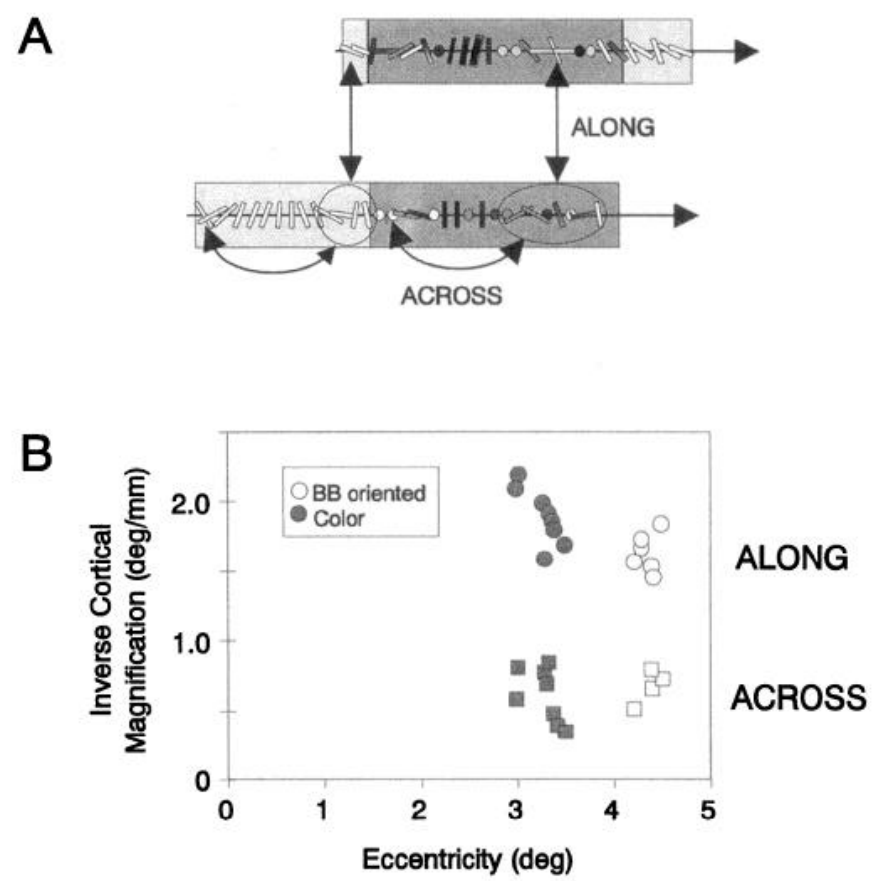

Figure 22. Anisotropy of V2 maps. A, Illustration of how comparison of cortical magnification was calculated across vs along stripes in a single case (for color detail see Fig. 4). Two parallel electrode penetrations which were made $1 \mathrm{~mm}$ apart on the cortical surface across a pale and a color stripe in V2. We calculated inverse cortical magnifications between a group of cells in each stripe (circled) with a cell $1 \mathrm{~mm}$ distant along the stripe and a second cell $1 \mathrm{~mm}$ distant across the same stripe. $B$, Results of this calculation. Inverse cortical magnification factors calculated across stripes are indicated by squares and those along stripes by circles; pale and color stripe calculations are indicated by open and solid symbols, respectively. The calculated cortical magnification factors ( $\mathrm{mm} /$ degree) along stripes are two to three times smaller than those across stripes; this is true for both the pale and the color stripes. 
penetration in this study, and estimation following flattening and reconstruction of brain), we cannot be confident about the accuracy of comparisons in magnification factors between these studies.

This overall magnification factor in V2 is also within the range of magnification factors reported in the $2^{\circ}-5^{\circ}$ eccentricity of V1 $\left(\sim 2^{\circ}-5 \mathrm{~mm} /\right.$ degree) (see also Daniel and Whitteridge, 1961; Hubel and Wiesel, 1974; Dow et al., 1981; Van Essen et al., 1984). However, in comparing magnifications between V1 and $\mathrm{V} 2$, it is important to separate the two axes of representation. As would be predicted by the reflection of the visual field across the V1/V2 border, overall cortical magnification in the axis across stripes in $V 2$ may be similar to that in VI. Due to the relative compression of the visual map along stripes in V2, cortical magnification along stripes should be much less than that in V1 (see Anisotropy of Map below).

Since there are twice as many pale as color or disparity stripes, one might have expected cortical magnification in pale stripes to be somewhat higher. However, that is not the case: cortical magnification in pale stripes is not significantly different from that in color stripes. Coupled with the fact that color stripes are on average twice the width of pale stripes (as indicated by the length of recording sequences in each stripe), this argues that the amount of cortex devoted to broad-band orientation representation is similar to that of color representation. In other words, on average, color and disparity stripes are twice as wide as pale stripes.

Why not three separate cortical areas?

The functional segregation in V2, which was initially suggested by anatomical staining and connectivity, has subsequently been confirmed by physiological characterization of receptive field types. Now this idea is further strengthened by our findings of topographic re-representation and independent receptive field size, scatter, and magnification factor within the different V2 stripe compartments. Furthermore, the reversal of topographic polarity in some disparity stripes is reminiscent of topographic reversals found at areal boundaries. How does this apparent degree of topographic and functional independence of stripes in V2 affect its status as a single cortical area? What are the possible advantages of interleaving the three distinct maps within V2?

We suggest that the multiple, interleaved mapping architecture in V2 is ideally suited for interstripe interactions, which could contribute to local perceptual binding. We suggest that the purpose of coalescing V1 inputs from one functional domain into a single stripe structure may be to generate higher order receptive field properties (such as spot cells or illusory contour cells which are likely to receive convergent inputs from across several degrees of space, cf. Hubel and Livingstone, 1985; Peterhans and Von der Heydt, 1993). The purpose of coalescing functionally disparate stripes representing the same region of space into a single cortical region (i.e., one stripe cycle) may be to ensure topographic alignment of multiple maps, thereby allowing the binding of these functionally distinct maps via local horizontal connections and the generation of coherent percepts (cf. Cavanagh, 1987).

Several lines of evidence suggest that such functional binding could take place via horizontal connections within V2. Injections of anatomical tracers into V2 stripes revealed patches of label contained primarily within the injected stripe cycle (i.e., within $4 \mathrm{~mm}$ or the injection site (Amir et al., 1993; Levitt et al., 1994).
Furthermore, these patches occurred both within the stripe injected and within each of the other two stripes types (Ts'o et al., 1990b; Levitt et al., 1994; Malach et al., 1994). Consistent with and adding to the anatomical findings, functional studies using cross correlation analysis also demonstrate interactions between different stripe compartments in V2 up to a distance of several stripes (Roe and Ts'o, 1992). Some of these interactions are quite specific with respect to receptive field properties. Both anatomical and physiological evidence provide a solid substrate by which the separate maps in V2 may be bound together. The functional and topographic architecture in V2 is thus suggestive of its role in the initial stages of visual perceptual binding.

\section{Modular units of topography in V2}

It has been shown by several investigators that stripes are actually composed of clusters or patches of staining. This has been demonstrated with cytochrome oxidase, anatomical tracers (Horton, 1984; Livingstone and Hubel, 1984; Wong-Riley, 1993), 2-deoxyglucose studies (Tootell and Hamilton., 1989), optical imaging (Ts'o et al., 1990b, 1991, 1994), and has been correlated with clustering of functional properties when studied electrophysiologically (Ts'o et al., 1990b, 1991). Anatomically, patchiness has been observed in connectivity from V1 (Horton, 1984; Livingstone and Hubel, 1984; Rockland and Virga, 1990), from the pulvinar (Curcio and Harting, 1978; Horton, 1985), from V4 (DeYoe et al., 1988), and MT (Zeki and Shipp, 1989; Krubitzer and Kaas, 1989), as well as from intra-areal sources (Rockland, 1985; Levitt et al., 1992). These clusters, which are about 0.5 $\mathrm{mm}$ in diameter, are larger than either blobs or orientation columns in V1 and appear in all three stripe domains. In the thin stripes, these are likely to represent color processing modules, such as those specific for red-green or blue-yellow opponency (e.g., Fig. 18; Ts'o et al., 1990b) or luminance modules characterized by broad-band unoriented cells (e.g., Fig. 15). Orientation modules in pale stripes have been shown to contain cells with similar orientation specificity and, unlike Vl where orientation changes continuously across cortex, are adjacent to modules of significantly different orientation selectivity (Ts'o et al., 1991; Fig. 3B). Disparity stripes have been shown to be characterized by clusters of near, far, tuned-excitatory, tuned-inhibitory cells (Ts'o et al., 1991), and may also contain domains where interactions with the color system take place (e.g., Fig. 19).

In this article, we have provided yet another feature by which functional modules in V2 can be defined. We have shown possible substripe topographies by demonstrating remapping within single in color stripes (Figs. 15-18) and disparity stripes (Fig. 19). By relating such repeated mappings to stripe substructures, we have demonstrated a significant relationship between functional clusters and topographic clusters. We suggest that the modules defined by anatomical and functional criteria also comprise the basic unit of topographic organization in $V 2$.

This notion is further supported by the curious finding that the axis of receptive field progression can be quite different from one cluster to the next. While dramatic changes in progression axis are rare for either the color or the pale stripes (although see pale stripe in Fig. 8), we have found that disparity clusters are consistently mapped in a reversed progression. These reversals in receptive field progression suggest that the visual map is set up independently in each of the stripe compartments (and subcompartments). While the significance of these reversals is unknown, they suggest that disparity stripes are closely tied in a 
topographic mirror-image manner to adjacent stripes or clusters. These issues can be further addressed with either anatomical (cf. Rockland et al., 1985; Ts'o et al., 1991; Levitt et al., 1994b) or functional (cf. Ts'o et al., 1993) connectivity studies within V2.

It is less clear whether pale stripes also support multiple maps. It is possible, given the general narrowness of pale stripes, that they might not support further subcompartmentalization, at least in the dimension crossing stripes. Our data contain only one example consistent with two receptive field progressions within a single pale stripe (Fig. 8). In this case, the intrastripe discontinuity occurs where there is no obvious functional border. However, it is important to bear in mind that to date we have only examined stripe substructure with respect to a few functional parameters, such as color versus luminance modulation. Indeed, there may be functional clustering in V2 stripes along functional dimensions which we have not yet begun to probe which would be well correlated with topographic clustering.

\section{The hypercolumn and point representations}

The term "hypercolumn" is often misunderstood and misused. Hubel and Wiesel (1974) introduced the concept of a hypercolumn as "a complete array of columns as a small machine that looks after all values of a given variable." Thus, an orientation hypercolumn is a complete set of orientation columns that span the full range of $180^{\circ}$ of orientation. Similarly, an ocular dominance hypercolumn is a set of left and right eye dominance columns. A common misconception identifies the hypercolumn with the classical illustration of the "ice cube" model of striate cortex (Hubel and Wiesel, 1974b), depicting the tidy registration of an ocular dominance hypercolumn and an orientation hypercolumn within a $1 \mathrm{~mm} \times 1 \mathrm{~mm}$ block of V1. Instead, we introduce the term point set to refer to this minimal set of hypercolumns, one for each of the represented functional dimensions, all dealing with a common point in visual space. The concept of the point set is also distinct from that of the point image, which factors in the aggregate receptive field size. Thus, while the point set occupies roughly a $1 \mathrm{~mm}$ block, Hubel and Wiesel observed that "containcd in a $2 \mathrm{~mm} \times 2 \mathrm{~mm}$ block there is more than enough machinery to digest ... a region of visual field (the aggregate size) ... in all orientations and with both eyes."

The interleaved nature of the ocular dominance and orientation systems in V1, their coexistence in single cells, and the similarity in size between the two types of hypercolumns made it easy to confuse the usage of the term "hypercolumn." $\mathrm{Al}$ though the cytochrome oxidase-rich blobs, a more recently introduced architectural feature in V1, are involved in color processing, it is unclear what might constitute a color hypercolumn. In particular, it is not yet clear whether a color hypercolumn conforms to the $1 \mathrm{~mm} \times 1 \mathrm{~mm}$ block that constitutes the point set defined in terms of ocular dominance and orientation in V1. Recent mapping studies in the V1 blobs (Landisman et al., 1994) have suggested that at parafoveal eccentricities, the color hypercolumn (at least for the red/green domain) is likely to be contained within $1 \mathrm{~mm}^{2}$ of $\mathrm{V} 1$.

The distinction between the concept of the hypercolumn and the point set is seen more dramatically in our current understanding of the functional architecture of V2. Unlike the ocular dominance and orientation hypercolumns of $\mathrm{V} 1$, the color, form, and disparity domains of $\mathrm{V} 2$ are represented in differing regions of cortex, in separate classes of cells. A color hypercolumn in V2 is contained in a thin stripe, and similarly for an orientation hypercolumn (pale stripe) or a disparity hypercolumn (thick stripe). The point set in V2 spans all three stripe types and is therefore distinct from a hypercolumn in V2. Analogous to the $2 \mathrm{~mm} \times 2 \mathrm{~mm}$ block of $\mathrm{Vl}$, aggregate receptive field sizes in V2 (cf. Fig. 9D of Rosa et al., 1989) suggest that the V2 point image would span two to three point sets in V2. Indeed, our recent optical imaging studies of point image in V2 confirm this notion (Roe and Ts'o, 1994).

\section{Anisotropy of map}

Our study suggests that the point set in V2 is quite anisotropic, being elongated in the dimension across stripes (isopolar dimension) and compressed in the dimension along stripes (isoeccentric dimension). Anisotropy has also been reported for the visuotopic map in Cebus monkey V2 (Rosa et al., 1988) as well as with respect to intracortical connectivities demonstrated anatomically (Rockland, 1985; Levitt et al., 1994b). A direct interpretation of our data would suggest that the dimensions of the point set is on the order of $6: 1$. If the map in V2 were the result of interleaving three separate isotropic maps, then one would expect the cortical magnification factor to triple in the dimension across the stripes. That is, in the isopolar axis three times as much cortex would be devoted to representing each degree of visual space than in the isoeccentricity axis. However, what we find is that the magnification across each stripe, at least across each of the pale and color stripes, is two times that along (Fig. 22 ), resulting in an overall expansion of six times across versus along the stripes. We suggest that this doubling reflects the remapping of visual space within stripe subcompartments. This doubling again supports the idea that the fundamental topographic unit is of the size of a functional cluster, roughly half the width of a color or disparity stripe width.

While the above reasoning follows directly from our data, one must bear in mind the possibility that there is further remapping in the dimension along the stripes. If so, then the map within single stripes may still be isotropic. In this case, the overall anisotropy ratio would drop to 3:1. Rosa et al. (1988) reported an anisotropic representation in Cebus V2, with magnification factor in the isopolar dimension bcing approximatcly 1.5 times that in the isoeccentric dimension. Besides possible species differences and differences in estimation methods, there could be local (e.g., central/peripheral) differences in degree of anisotropy which could contribute to this discrepancy.

\section{Conclusion}

Important transformations in organizational strategy lake place as one ascends the cortical hierarchy. In this article, we have revealed a significant change in topographic mapping strategy. The repeated and interleaved maps in V2 are an illustration of how, with ascending cortical hierarchy, organization with respect to functional stimulus features such as color, orientation, and disparity begin to take precedence over simple, faithful visuotopic mapping. Unlike the relatively orderly retinotopy of the lateral geniculate nucleus or V1, topography in V2 is parcellated into functional modules which are locally retinotopic. The size of these V2 modules is greater than the orientation modules and blobs of $\mathrm{V} 1$, suggesting that each area may have a characteristic module size which increases with cortical hierarchy (cf. Amir et al., 1993; Ghose et al., 1994). Collections of these modules spanning a particular functional property constitute a hypercolumn. Unlike the orientation and ocular dominance hypercolumns of $\mathrm{V} 1$, however, the hypercolumns in V2 are spatially segregated. The collection of hypercolumns for a point in space, which we 
define as the point set, spans the width of an entire stripe cycle (thin/pale/thick/pale) in V2. The interplay between the maintenance of global retinotopy and more local functional specialization has created a complex and rich functional and topographic architecture in V2 that must profoundly reflect its role in visual processing.

\section{References}

Albright TD, Desimone R (1987) Local precision of visuotopic organization in the middle temporal area of the macaque. Exp Brain Res 65:582-592.

Allman JM, Kaas JH (1974) The organization of the second visual area (V-II) in the owl monkey: a second order transformation of the visual hemifield. Brain Kes $16: 24 \%-265$.

Amir Y, Harel M, Malach R (1993) Cortical hierarchy reflected in the organization of intrinsic connections in macaque monkey visual cortex.

Baizer JS, Robinson DL, Dow BM (1977) Visual responses of area 18 neurons in awake, hehaving monkey. I Neurophysiol 40:1024-1037.

Blasdel GG, Fitzpatrick D (1984) Physiological organization of layer 4 in macaque striate cortex. J Neurosci 4:880-895.

Cavanagh P (1987) Multiple analyses of orientation in the visual sys tem. In: Neural mechanisms of visual perception (Lam DM, Gilbert CD, eds), pp 261-279. Texas: Portfolio.

Curcio CA, Harting JK (1978) Organization of pulvinar afferents to area 18 in the squirrel monkey: evidence for stripes. Brain Res 143: 155-161.

Daniel PM, Whitteridge D (1961) The representation of the visual field in the cerebral cortex in monkeys. J Physiol (Lond) 159:302-321.

DeYoe EA, Van Essen DC (1985) Segregation of efferent connections and receptive field properties in visual area V2 of the macaque. Nature 317:58-61.

DeYoe EA, Van Essen DC (1988) Concurrent processing streams in monkey visual cortex. Trends Neurosei 11:219-226.

DeYoe EA, Felleman DJ, Knierim JJ, Olavarria JF, Van Essen DC (1988) Heterogeneous subregions of macaque visual area $V A$ receive selective projections from V2 thin-stripe and interstripe subregions. Invest Ophthalmol Vis Sci [Suppl] 29:115.

DeYoe EA, Hockfield S, Garren H, Van Essen DC (1990) Antibody labeling of functional subdivisions in visual cortex: Cat-301 immunoreactivity in striate and extrastriate cortex of the macaque monkey. Vis Neurosci 5:67-8I.

Dow BM, Snyder AZ, Vautin RG, Bauer R (1981) Magnification factor and receptive field size in foveal striate cortex of the monkey. Exp Brain Res 44:213-228.

Frostig RD, Lieke EE, Ts'o DY, Grinvald AM (1990) Cortical functional architecture and local coupling between neuronal activity and the microcirculation revealed by in vivo high-resolution optical imaging of intrinsic signals. Proc Natl Acad 3ci USA 87:6082-6086.

Gattas R, Gross CG (1981) Visual topography of the striate projection zone in the posterior superior temporal sulcus (MT) of the macaque. J Neurophysiol 46:621-637.

Gatlass R, Gross CG, Sandell JH (1981) Visual topography of V2 in the Macaque. J Comp Neurol 201:519-539.

Ghose GM, Roe AW, Ts'o DY (1994) Features of functional organization within primate V4. Soc Neurosci Abstr 20:840.

Grinvald A, Lieke E, Frostig RD, Gilbert CD, Wiesel TN (1986) Functional architecture of cortex revealed by optical imaging of intrinsic signals. Nature 324:361-364.

Grinvald A, Frostig RD, Lieke E, Hildesheim R (1988) Optical imaging of neuronal activity. Physiol Rev 68:1285-1365.

Horton J (1984) Cytochrome oxidase patches: a new cytoarchitectonic feature of monkey visual cortex. Philos Trans R Soc Lond [Biol] 304: 199.253.

Horton J, Hubel (1981) A regular patchy distribution of cytochromeoxidase staining in primary visual cortex of the macaque monkey. Nature 292:762-764.

Hubel DH, Livingstone MS (1985) Complex-unoriented cells in a subregion of primate area 18 . Nature 315:325-327.

Hubel DH, Livingstone MS (1987) Segregation of form, color, and stereopsis in primate area 18. J Neurosci 7:3378-3415.

Hubel DH, Wiesel TN (1970) Cells sensitive to binocular depth in area 18 of the macaque monkey cortex. Nature 225:41-42.

Hubel DH, Wiesel TN (1974) Uniformity of monkey striate cortex: a parallel relationship between field size, scatter, and magnification factor. J Comp Neurol 158:295-305.

Hubel DH, Wiesel TN (1977) Functional architecture of macaque monkey visual cortex. Proc R Soc Lond [Biol] 198:1-59.

Hubel DH, Wiesel TN, LeVay S (1974) Visual field representation in layer IVC of monkey striate cortex. Soc Neurosci Abstr 4:264.

Irnig TJ, Brugge JF (1978) Sources and terminations of callosal axons related to binaural and frequency maps in primary auditory cortex of the cat. J Comp Neurol 182:637-660.

Kaas JH (1987) The organization of neocortex in mammals: implications for theories of brain function. Annu Rev Psychol 38:129-151.

Krubitzer LA, Kaas JH (1989) Cortical integration of parallel pathways in the visual system of primates. Brain Res 478:161-165.

Krubitzer LA, Kaas JH (1990a) Cortical connections of MT in four species of primates: areal, modular, and retinotopic patterns. Vis Neurosci 5:165-204.

Krubitzer L, Kaas (1990b) Convergence of processing channels in the extrastriate cortex of monkeys. Vis Neurosci 5:609-613.

Landisman CE, Roe AW, Ts'o DY (1994) The relationship of receptive field coverage to functional modules in primate V1. Soc Neurosci Abstr 20:1477.

Levitt JB, Kiper DC, Movshon JA (1994a) Receptive fields and functional architecture of macaque V2. J Neurophysiol 71:2517-2542.

Levitt JB, Yoshioka T, Lund JS (1994b) Intrinsic cortical connections in macaque visual area V2: evidence for interaction between different functional streams. J Comp Neurol 342:551-570.

Lieke ED, Frostig RD, Arieli A, Ts'o DY, Hildesheim R, Grinvald A (1989) Optical imaging of cortical activity: real-time imaging using extrinsic dye-signals and high resolution imaging based on slow intrinsic signals.

Livingstone MS, Hubel DH (1984) Anatomy and physiology of a color system in the primate visual cortex. J Neurosci 4:309-356.

Livingstone MS, Hubel DH (1987a) Connections between layer 4B of area 17 and the thick cytochrome oxidase stripes of area 18 in the squirrel monkey. J Neurosci 7:3371-3377.

Livingstone MS, Hubel DH (1987b) Psychophysical evidence for separate channels for the perception of form, color, and movement, and depth. J Neurosci 7:3416-3468.

Malach R, Tootell RBH, Malonek D (1994) Relationship between orientation domains, cytochrome oxidase stripes, and intrinsic horizontal connections in squirrel monkey area V2. Cereb Cortex 4:151-165.

Middlebrooks JC, Dykes RW, Merzenich MM (1980) Binaural response-specific bands in primary auditory cortex (AI) of the cat: topographical organization orthogonal to isofrequency contours. Brain Res 181:31-48

Morgan MJ, Aiba TS (1985) Positional acuity with chromatic stimuli. Vision Res 25:689-695.

Nakamura H, Gattass R, Desimone R, Ungerleider LG (1993) The modular organization of projections from areas V1 and V2 to areas V4 and TEO in macaques. J Neurosci 13:3681-3691.

Peterhans E, von der Heydt R (1989) Mechanisms of contour perception in monkey visual cortex. II. Contours bridging gaps. J Neurosci 9:1749-1763.

Peterhans E, von der Heydt R (1993) Functional organization of area $\mathrm{V} 2$ in the alert macaque. Eur $\mathbf{J}$ Neurosci 5:509-524.

Poggio GF, Fischer B (1977) Binocular interaction and depth sensitivity in striate and prestriate cortex of behaving rhesus monkey. $\mathbf{J}$ Neurophysiol 40:1392-1405.

Rockland KS (1985) A reticular pattern of intrinsic connections in primate area V2 (area 18). J Comp Neurol 235:467-478.

Rockland KS, Virga A (1990) Organization of individual cortical axons projecting from area V1 (area 17) to V2 (area 18) in the macaque monkey. Vis Neurosci 4:11-28.

Roe AW, Ts'o DY (1992) Functional connectivity between V1 and V2 in the primate. Soc Neurosci Abstr 18:11.

Roe AW, Ts'o DY (1993a) Visual field representation within primate V2 and its relationship to the functionally distinct stripes. Invest Ophthalmol Vis Res 34:812.

Roe AW, Ts'o DY (1993b) Visual representation and functional compartments within single V2 stripes. Soc Neurosci Abstr 19:1499.

Roe AW, Ts'o DY (1994) Relationships between topographic maps in V1 and V2 revealed by optical imaging with spot stimuli. Soc Neurosci Abstr 20:840.

Rosa MGP, Sousa APB, Gattass R (1988) Representation of the visual 
field in the second visual area in the Cebus monkey. $J$ Comp Neurol 275:326-345.

Shipp S, Zeki S (1985) Segregation of pathways leading from area V2 to areas V4 and V5 of macaque monkey. Nature 315:322-325.

Shipp S, Zeki S (1989) The organization of connections between areas V5 and V2 in macaque monkey visual cortex. Eur J Neurosci 1:333354.

Sretevan D, Dykes RW (1983) The organization of two cutaneous submodalities in the forearm region of area $3 \mathrm{~b}$ of cat somatosensory cortex. J Comp Neurol 213:381-398.

Sur M, Wall JT, Kaas JH (1981) Modular segregation of functional cell classes within the postcentral somatosensory cortex of monkeys. Science 212:1059-1061.

Tootell RBH, Hamilton SL (1989) Functional anatomy of the second visual area (V2) in the Macaque. J Neurosci 9:2620-2644.

Tootell RBH, Silverman MS, De Valois RL, Jacobs GH (1983) Functional organization of the second cortical visual area in primates. Science 220:737-739.

Tootell RBH, Hamilton SL, Silverman MS (1985) Topography of cytochrome oxidase activity in the owl monkey cortex. J Neurosci 5:2786-2800.

Ts'o DY, Gilbert CD (1988) The organization of chromatic and spatial interactions in the primate striate cortex. J Neurosci 8:1712-1727.

Ts'o DY, Roe AW (1994) Functional compartments in visual cortex: segregation and interaction. In: The cognitive neurosciences (Gazzaniga MS, ed), pp 325-337. Cambridge, MA: MIT Press.

Ts'o DY, Gilbert CD, Frostig RD, Grinvald A, Wiesel TN (1989) Functional architecture of visual area 18 of macaque monkey. Soc Neurosci Abstr 15:161

Ts'o DY, Frostig RD, Lieke EE, Grinvald A (1990a) Functional organization of primate visual cortex revealed by high resolution optical imaging. Science $249: 417-420$.

Ts'o DY, Gilbert CD, Wiesel TN (1990b) Functional architecture of color and disparity in visual area 2 of macaque monkey. Soc Neurosci Abstr 16:293.
Ts'o DY, Gilbert CD, Wiesel TN (1991) Orientation selectivity of and interactions between color and disparity subcompartments in area V2 of Macaque monkey. Soc Neurosci Abstr 17:1089.

Ts'o DY, Roe AW, Shey J (1993) Functional connectivity within V1 and V2: patterns and dynamics. Soc Neurosci Abstr 19:1499.

Van Essen DC (1985) Functional organization of primate visual cortex. In: Cerebral cortex, Vol 3 (Jones EG, Peters AA, eds), pp 259-329. New York: Plenum.

Van Essen DC, Zeki SM (1978) The topographic organization of rhesus monkey prestriate cortex. J Physiol (Lond) 277:193-226.

Van Essen DC, Newsome WT, Maunsell JHR (1984) The visual field representation in striate cortex of the macaque monkey: asymmetries, anisotropies, and individual variability. Vision Res 24:429-448.

Van Essen DC, Newsome WT, Maunsell JHR, Bixby JL (1986) The projections from striate cortex (V1) to areas V2 and V3 in the macaque monkey: asymmetries, areal boundaries, and patchy connections. J Comp Neurol 244:451-480.

Weller RE, Kaas JH (1983) Retinotopic patterns of connections of area 17 with visual areas V-II and MT in Macaque monkeys. J Comp Neurol 220:253-279.

Wong-Rilcy MTT (1979) Changes in the visual system of monocularly sutured or enucleated cats demonstrable with cytochrome oxidase histochemistry. Brain Res 171:11-28.

Wong-Riley MTT, Hevner RF, Cutlan R, Earnest M, Egan R, Frost J, Nguyen T (1993) Cytochrome oxidase in the human visual cortex: distribution in the developing and the adult brain. Vis Neurosci 10: $41-58$.

Zeki S (1990) Functional specialization in the visual cortex: the generation of separate constructs and their multistage integration. In: Signal and sense local and global order in perceptual maps (Edelman GM, Gall WE, Cowan WM, eds). New York: Wiley-Liss.

Zeki S, Shipp S (1987) Functional segregation within area V2 of macaque monkey visual cortex. In: Seeing contour and color (Kulikowski JJ, Dickinson CM, Murray IJ, eds), pp 120-124. Oxford: Pergamon. 